\title{
DETERMINATION OF THE ELECTROWEAK CHIRAL-LAGRANGIAN PARAMETERS AT THE LHC
}

\author{
A. Dobado \\ Departamento de Física Teórica \\ Universidad Complutense de Madrid \\ 28040 Madrid, Spain \\ and \\ M. T. Urdiales q \\ Departamento de Física Teórica \\ Universidad Autónoma de Madrid \\ 28049 Madrid, Spain
}

February 1995

\begin{abstract}
In this work we report on the results obtained in a detailed and systematical study of the possibility to measure the parameters appearing in the electroweak chiral lagrangian. The main novelty of our approach is that we do not use the Equivalence Theorem and therefore we work explicitly with all the gauge boson degrees of freedom.
\end{abstract}

hep-ph/9502255

\footnotetext{
${ }^{1}$ E-mail: dobado@cernvm.cern.ch

${ }^{2}$ E-mail: mayte@vm1.sdi.uam.es
} 


\section{Introduction}

Today it is clear for many physicists that one of the main goals of the future CERN Large Hadron Collider $(L H C)$ is to find as much information as possible about the nature of the Standard Model $(S M)$ Electroweak Symmetry Breaking $(E S B)$ [1]. In spite of the huge amount of data obtained in the last years at the Large Electron-Positron Collider $(L E P)$ it is very few what we really know about the ESB. The proposed mechanisms include ideas such as supersymmetry (see [2] and references therein), technicolor [3] and many others. Therefore it would be very interesting to have some model independent framework to make a phenomenological description of the ESB physics.

In fact such a framework exists, at least for the strongly interacting case i.e., when no light modes are present in the ESB. It has been developed in the last years and used to describe the scattering of the longitudinal components $(L C)$ of the electroweak gauge bosons [4] as well as the precision tests of the Standard Model coming from LEP [5]. It is based on the application of the chiral Lagrangians or Chiral Perturbation Theory $(\chi P T$ )[6] (previously invented for the description of the low-energy hadron interactions) to the dynamics of the ESB Goldstone bosons $(G B)$.

One assumes that there is a physical system with a global symmetry group $G$ which is spontaneously broken to some other group $H$. This global symmetry breaking drives the gauge electroweak $S U(2)_{L} \times U(1)_{Y}$ symmetry breaking down to $U(1)_{e m}$ through the well known Higgs mechanism. The only election for the $G$ and $H$ groups compatible with the presence of the $S U(2)_{L+R}$ custodial symmetry [7] (to have a $\rho$ parameter naturally close to one) and the existence of three massive gauge bosons after the $E S B$ i.e., the $W^{+}, W^{-}$ and $Z^{0}$, is $G=S U(2)_{L} \times S U(2)_{R}, H=S U(2)_{L+R}$.

Then the low energy dynamics of the $E S B$ is described by a $S U(2)_{L} \times U(1)_{Y}$ gauged non-linear sigma model $(G N L S M)$ including an arbitrary large number of terms in the action with different number of derivatives of the $G B$ fields and electroweak gauge bosons. The corresponding couplings (parameters) encode the dynamics of the ESB sector of the $S M$ and must be renormalized to absorb divergences. However, at low enough energies only a small number of terms (and couplings) are needed to eliminate all the divergences. In principle the values of these couplings or parameters could be obtained from the underlying theory or directly by fitting them from future experiments.

In this work we will study the possibilities of the $L H C$ for measuring these parameters and the expected corresponding errors. In particular we will concentrate in events producing $Z^{0} Z^{0}$ or $W^{ \pm} Z^{0}$ pairs. As it was mentioned above, the application of the chiral Lagrangian technique to the production of electroweak gauge bosons is not new and has already been considered at the literature [8]. However, all the applications worked out until now are based in the so called Equivalence Theorem (ET) [9]. This theorem relates the $S$ matrix elements of processes containing electroweak gauge bosons $L C$ with the corresponding processes with $G B$. However, in a recent work [10 concerning the formulation of the ET in the context of $\chi P T$, the Equivalence Theorem is severely restricted to 
a narrow energy applicability window. It can also be applied in the high energy domain together with the $\chi P T$ but using some non-perturbative technique, like dispersion relations [11] or the large $N$ limit [12], in order to have an appropriate unitarity behaviour of the amplitudes.

For this reason we consider prioritary to apply directly the chiral Lagrangian description of the $E S B$ without using the ET. The main problems of this approach are two: First one has to include explicitly the gauge degrees of freedom in the model which makes the computations extremely more difficult. Second, one has to restrict the results to the low energy region where standard $\chi P T$ can safely be applied thus losing many higher energy events. The advantage is that the values of the fitted parameters will be more reliable since one is not using the ET complemented with some non-perturbative method.

The plan of this work goes as follows: In section 2 we introduce the chiral effective lagrangian to be used with its parameters and the corresponding Feynman rules. In section 3 we compute the cross-section of the $L H C$ subprocesses that are relevant for measuring the parameters. In section 4 we consider the signatures and the possible backgrounds. In section 5 we show how we compute the total number of expected events at the $L H C$ from the subprocess cross-sections for the signals and backgrounds. In section 6 we discuss the sensitivity of the machine to the different parameters, we define the optimal cuts and we compute the statistical significance of the different parameter measures and estimate the statistical errors. In section 7 we consider other sources of systematical errors such as the uncertainty on the proton structure functions and in section 8 the effect of the running of the parameters. Finally, in section 9 we review the main conclussions of our work.

\section{The effective Lagrangian and the Feynman rules}

In this section we consider the most general effective Lagrangian for the $E S B$ compatible with the $S U(2)_{L} \times U(1)_{Y}$ local symmetry of the $S M$ and the breaking pattern $S U(2)_{L} \times$ $U(1)_{Y} \rightarrow U(1)_{\mathrm{em}}$. The well known chiral Lagrangian describing the $E S B$ of the $S M$, can be written as an infinite expansion with terms of increasing number of gauge fields and derivatives of the Goldstone bosons $(G B)$, with an infinite number of arbitrary parameters. This chiral Lagrangian can be seen as a low momentum expansion for the corresponding Green functions. At some given order in the number of $G B$ derivatives one can work only with a finite number of terms and parameters. In this case the model can only be applied to much smaller energies than $4 \pi v$ which is the parameter controlling this expansion $(4 \pi v \simeq 3 T e V$ since $v \simeq 250 \mathrm{GeV})$.

The first term of this effective Lagrangian $\left(O\left(p^{2}\right)\right)$ is given by:

$$
\mathcal{L}^{(2)}=\frac{v^{2}}{4} \operatorname{tr}\left[D_{\mu} U\left(D^{\mu} U\right)^{\dagger}\right]
$$


and by the Yang-Mills Lagrangian:

$$
\mathcal{L}_{Y M}=-\frac{1}{4} B_{\mu \nu} B^{\mu \nu}-\frac{1}{2} \operatorname{Tr}\left[F_{\mu \nu} F^{\mu \nu}\right]
$$

We choose the GB parametrization as an unitary matrix $U$ belonging to the quotient space $S U(2)_{L} \times S U(2)_{R} / S U(2)_{L+R}$.

$$
U=\exp \left(i \frac{\vec{\tau} \cdot \vec{\pi}}{v}\right)
$$

where $\vec{\tau} \equiv\left(\tau_{1}, \tau_{2}, \tau_{3}\right)$ are the Pauli matrices and $\vec{\pi} \equiv\left(\pi_{1}(x), \pi_{2}(x), \pi_{3}(x)\right)$ represents the triplet of $G B$.

The covariant derivative $D_{\mu} U$ is defined as:

$$
D_{\mu} U=\partial_{\mu} U+i g W_{\mu} U-i g^{\prime} U Y_{\mu}
$$

Here, $W_{\mu}$ and $Y_{\mu}$ are the $S U(2)_{L}$ and the $U(1)_{Y}$ gauge fields given by:

$$
\begin{gathered}
W_{\mu}=\frac{\vec{W}_{\mu} \vec{\tau}}{2} \\
Y_{\mu}=\frac{B_{\mu} \tau_{3}}{2}
\end{gathered}
$$

where $W_{\mu}=\left(W_{\mu}^{1}, W_{\mu}^{2}, W_{\mu}^{3}\right)$ represents the triplet of $S U(2)_{L}$ gauge fields.

As usual, the covariant field strength tensors are defined as

$$
\begin{aligned}
& F_{\mu \nu}(x)=\partial_{\mu} W_{\nu}(x)-\partial_{\nu} W_{\mu}(x)+i g\left[W_{\mu}(x), W_{\nu}(x)\right] \\
& B_{\mu \nu}(x)=\partial_{\mu} B_{\nu}(x)-\partial_{\nu} B_{\mu}(x)
\end{aligned}
$$

The transformation properties under the $S U(2)_{L} \times U(1)_{Y}$ gauge transformations are

$$
\begin{aligned}
i g W_{\mu}^{\prime} & =g_{L}(x) i g W_{\mu} g_{L}^{\dagger}(x)+g_{L}(x) \partial_{\mu} g_{L}^{\dagger}(x) \\
i g^{\prime} Y_{\mu}^{\prime} & =i g^{\prime} Y_{\mu}+g_{Y}(x) \partial_{\mu} g_{Y}^{\dagger}(x) \\
U^{\prime} & =g_{L}(x) U g_{Y}^{\dagger}(x)
\end{aligned}
$$

where 


$$
\begin{aligned}
& g_{L}(x)=e^{i \theta_{k}(x) \tau^{k} / 2} \\
& g_{Y}(x)=e^{i \beta(x) \cdot \tau^{3} / 2}
\end{aligned}
$$

Note that, as expected, the $G B$ fields, $\pi_{i}$, transform non-linearly .

In order to construct the chiral lagrangian to order $O\left(p^{4}\right)$ we define, following Longhitano in [13], the quantities $T, V_{\mu}$ and $\mathcal{D}_{\mu} O(x)$ :

$$
\begin{aligned}
T & =U \tau^{3} U^{\dagger} \\
V_{\mu} & =D_{\mu} U U^{\dagger} \\
\mathcal{D}_{\mu} O(x) & =\partial_{\mu} O(x)+i g\left[W_{\mu}(x), O(x)\right]
\end{aligned}
$$

Then the complete electroweak chiral Lagrangian with the whole set of $S U(2) \times U(1)_{Y}$, Lorentz, $C, P$ and $T$ invariant operators up to dimension four has the form:

$$
\mathcal{L}=\mathcal{L}^{(2)}+\mathcal{L}_{Y M}+\mathcal{L}^{\prime}{ }_{1}+\sum_{i=1}^{13} \mathcal{L}_{i}
$$

In this equation, the different terms $\mathcal{L}_{i}$ are the $S U(2)_{L} \times U(1)_{Y}$ invariant functions of the gauge vector bosons and the $G B$ fields, containing four derivatives, whereas $\mathcal{L}_{1}^{\prime}$ has dimension two. They have the following expressions:

$$
\begin{aligned}
\mathcal{L}^{\prime} & =\frac{1}{4} g^{2} \alpha_{0} v^{2}\left[\operatorname{Tr}\left(T V_{\mu}\right)\right]^{2} \\
\mathcal{L}_{1} & =\frac{1}{2} g^{2} \alpha_{1} B_{\mu \nu} \operatorname{Tr}\left(T F^{\mu \nu}\right) \\
\mathcal{L}_{2} & =\frac{1}{2} i g \alpha_{2} B_{\mu \nu} \operatorname{Tr}\left(T\left[V^{\mu}, V^{\nu}\right]\right) \\
\mathcal{L}_{3} & =i g \alpha_{3} \operatorname{Tr}\left(F_{\mu \nu}\left[V^{\mu}, V^{\nu}\right]\right) \\
\mathcal{L}_{4} & =\alpha_{4}\left[\operatorname{Tr}\left(V_{\mu} V_{\nu}\right)\right]^{2} \\
\mathcal{L}_{5} & =\alpha_{5}\left[\operatorname{Tr}\left(V_{\mu} V^{\mu}\right)\right]^{2} \\
\mathcal{L}_{6} & =\alpha_{6} \operatorname{Tr}\left[\left(V_{\mu} V_{\nu}\right)\right] \operatorname{Tr}\left(T V^{\mu}\right) \operatorname{Tr}\left(T V^{\nu}\right) \\
\mathcal{L}_{7} & =\alpha_{7} \operatorname{Tr}\left[\left(V_{\mu} V^{\mu}\right)\right]\left[\operatorname{Tr}\left(T V^{\nu}\right)\right]^{2} \\
\mathcal{L}_{8} & =\frac{1}{4} g^{2} \alpha_{8}\left[\operatorname{Tr}\left(T F_{\mu \nu}\right)\right]^{2} \\
\mathcal{L}_{9} & =\frac{1}{2} i g \alpha_{9} \operatorname{Tr}\left(T F_{\mu \nu}\right) \operatorname{Tr}\left(T\left[V^{\mu}, V^{\nu}\right]\right)
\end{aligned}
$$




$$
\begin{aligned}
\mathcal{L}_{10} & =\frac{1}{2} \alpha_{10}\left[\operatorname{Tr}\left(T V_{\mu}\right) \operatorname{Tr}\left(T V_{\nu}\right)\right]^{2} \\
\mathcal{L}_{11} & =\alpha_{11} \operatorname{Tr}\left[\left(\mathcal{D}_{\mu} V^{\mu}\right)^{2}\right] \\
\mathcal{L}_{12} & =\frac{1}{2} \alpha_{12} \operatorname{Tr}\left(T \mathcal{D}_{\mu} \mathcal{D}_{\nu} V^{\nu}\right) \operatorname{Tr}\left(T V^{\mu}\right) \\
\mathcal{L}_{13} & =\frac{1}{2} \alpha_{13}\left[\operatorname{Tr}\left(T \mathcal{D}_{\mu} V^{\nu}\right)\right]^{2}
\end{aligned}
$$

As it is well known there is some arbitrariness in the choice of a particular base of invariants. Using the equations of motion it would be possible to eliminate the operators $\mathcal{L}_{11}, \mathcal{L}_{12}$ and $\mathcal{L}_{13}$ redefining the rest of the terms (see for example [14]).

So, a set of $\alpha_{k}(k=0, . .13)$ parameters have appeared in the definition of this effective Lagrangian. We display in Table 1 the relations between some different sets of the chiral Lagrangian parameters used in the literature, i.e. by Longhitano [13], Feruglio [14] and Dobado et.al. [5].

The theory defined by the Lagrangian above is non-renormalizable in the strict sense, but the divergences appearing when we calculate at one-loop with the chiral Lagrangian at lowest order eq.(11) have the same form that some of the terms obtained at tree level in the following order in the chiral expansion. The counterterms needed to reabsorb the divergences generated to the one-loop level with $\mathcal{L}^{(2)}$ in the Landau gauge were obtained by the authors in [13]. They have the same form that $\mathcal{L}_{1}^{\prime}, \mathcal{L}_{1}, \mathcal{L}_{2}, \mathcal{L}_{3}, \mathcal{L}_{4}$ and $\mathcal{L}_{5}$, whereas the rest of the terms in eq.(4) are not needed for renormalization.

We will describe the interactions between gauge bosons and fermions by the same Lagrangian than that of the $S M$, which can also describe the couplings between fermions and scalars by means of the usual Yukawa terms. However the latter are not used in our calculations since we work all the time with massless fermions.

Once we have set the Lagrangian to be used, the next step is to define the quantum theory. This can be done in a standard way using the Faddeev-Popov method and choosing some appropriate $R_{\xi}$ covariant gauge. The gauge fixing functions are constructed by requiring that the terms of the form $W_{\mu}^{i} \partial^{\mu} \pi_{i}(x)$ and $B_{\mu} \partial^{\mu} \pi_{3}$ cancel out; thus yielding:

$$
\begin{aligned}
f_{i}\left(W_{i \mu}\right) & =\partial^{\mu} W_{i \mu}-\frac{v}{2} \xi_{W} g \pi_{i}, \quad i=1,2,3 \\
f_{0}\left(B_{\mu}\right) & =\partial^{\mu} B_{\mu}+\frac{v}{2} \xi_{B} g^{\prime} \pi_{3}
\end{aligned}
$$

Now we can write the gauge-fixing and Faddeev-Popov terms as:

$$
\mathcal{L}_{G F}=-\frac{1}{2 \xi_{W}} \sum_{i=1}^{3}\left[f_{i}\left(W_{i \mu}\right)\right]^{2}-\frac{1}{2 \xi_{B}}\left[f_{0}\left(B_{\mu}\right)\right]^{2}
$$




$$
\mathcal{L}_{F P G}=\int d^{4} y c^{\dagger}{ }_{m}(x) \frac{\delta f_{m}(x)}{\delta \theta_{n}(y)} c_{n}(y) \quad m, n=0,1,2,3
$$

Therefore, the effective action and the effective Lagrangian (non-linear $S M$ Lagrangian, $\left.\mathcal{L}_{N L S M}\right)$ take the form:

$$
\begin{gathered}
S_{\text {eff }}=\int d^{4} x\left[\mathcal{L}(x)+\mathcal{L}_{G F}+\mathcal{L}_{F P G}\right] \\
\mathcal{L}_{N L S M}=\mathcal{L}^{(2)}+\mathcal{L}^{\prime}{ }_{1}+\mathcal{L}^{(4)}+\mathcal{L}_{Y M}+\mathcal{L}_{G F}+\mathcal{L}_{F P G}+\mathcal{L}_{\Psi}
\end{gathered}
$$

In the following we will to work in the Landau gauge $\left(\xi_{W}=\xi_{B}=0\right)$. In this gauge the perturbative $\pi$ propagator remains massless and the Faddeev-Popov ghosts only couple to the gauge field. There is not direct coupling of the $\pi$ field to the ghosts. Moreover, the counterterms necessary to cancel divergences with $\mathcal{L}^{(2)}$ at one loop are gauge invariant functions of the gauge fields and the $G B$ alone (the $\mathcal{L}_{1}^{\prime}, \mathcal{L}_{1}, \mathcal{L}_{2}, \mathcal{L}_{3}, \mathcal{L}_{4}$ and $\mathcal{L}_{5}$ terms mentioned above). In other gauges, or other parametrizations of the coset space, the counterterms could also be functions of the ghost fields, and their structure would be determined using the Becchi-Rouet-Stora $(B R S)$ invariance.

Thus, the gauge fixing and the Faddeev-Popov term in the Landau gauge read:

$$
\begin{aligned}
\mathcal{L}_{G F} & =-\frac{1}{2 \xi} \sum_{i=1}^{3}\left[\partial^{\mu}\left(W_{i \mu}\right)\right]^{2}-\frac{1}{2 \xi}\left[\partial^{\mu}\left(B_{\mu}\right)\right]^{2} \\
\mathcal{L}_{F P G} & =\partial_{\mu} c_{i}^{\dagger} \partial^{\mu} c^{i}-g \epsilon_{i j k} c^{i \dagger} c^{j} \partial^{\mu} W_{\mu}{ }^{k}+\partial_{\mu} c_{0}^{\dagger} \partial^{\mu} c^{0}
\end{aligned}
$$

In the Appendix we show the Feynman rules derived from the Lagrangian $\mathcal{L}_{N L S M}$ eq.(7) that we use for our calculations. They correspond to Figures 1, 22 and 3. As usual it has been useful to redefine the gauge fields as:

$$
\begin{aligned}
W_{\mu}^{ \pm} & =\frac{W_{\mu}^{1} \mp i W_{\mu}^{2}}{\sqrt{2}} \\
Z_{\mu} & =\cos \theta_{W} W_{\mu}^{3}-\sin \theta_{W} B_{\mu} \\
A_{\mu} & =\sin \theta_{W} W_{\mu}^{3}+\cos \theta_{W} B_{\mu}
\end{aligned}
$$

where the weak angle is defined by

$$
\operatorname{tg} \theta_{W}=\frac{g^{\prime}}{g}
$$


and the $G B$ as:

$$
\begin{aligned}
& \pi^{ \pm}=\frac{\pi^{1} \mp i \pi^{2}}{\sqrt{2}} \\
& \pi^{0}=\pi^{3}
\end{aligned}
$$

\section{The subprocesses cross-sections}

In this work we are interested in studying of the different cross sections that contribute to the final states $Z^{0} Z^{0}$ and $W^{ \pm} Z^{0}$ at $p p$ colliders such as $L H C$ since they are the most promising from the experimental point of view. In both cases we focus our attention on the so-called gold-plated events, where the produced bosons decay to the leptonic final states $l=e, \mu, \nu$. In spite of the very small branching ratios $(B R)$,

$$
\begin{aligned}
B R\left(Z^{0} Z^{0} \rightarrow l \bar{l} l^{\prime} \overline{l^{\prime}}\right) & =0.0044 \\
B R\left(W^{ \pm} Z^{0} \rightarrow l \nu_{l} l^{\prime} \bar{l}^{\prime}\right) & =0.013
\end{aligned}
$$

these are the most interesting events since they are much easier to detect than the hadronic channels.

On the other hand, we will assume that it is not possible to detect experimentally the different polarizations of the gauge bosons in the final state. Therefore we have to include in our computations all the $Z^{0}$ or $W^{ \pm}$gauge bosons, either transversally or longitudinally polarized. As it was mentioned in the introduction, instead of using the ET to calculate the collisions of the weak bosons, as it is usually done, we have computed the amplitudes with gauge bosons as initial and final states and then projected them in all their polarizations. Thus, as we are not going to apply the $E T$ we only consider the maximal bound on the energy required for the applicability of the chiral Lagrangian formalism, but we do not have any low energy bound. In this way we can take into account the influence of the chiral lagrangian parameters in all the gauge bosons polarization channels and not only in the longitudinal ones as it is usually the case. The main disadvantage in our procedure is that the calculations become much more complicated. This fact leads us to work only at tree level to make the computation more accessible.

In principle, according to the spirit of $\chi P T$, the one-loop corrections coming from the lowest order lagrangian should also be included since they are $O\left(p^{4}\right)$. However, the computation of these corrections is extremely involved. In addition, they have not dependence on the chiral parameters. As it will become clear later, our main interest is to study the dependence of the number of events on the chiral parameters in order to see 
which of them could be measured at the $L H C$. For this reason we do not expect that our results concerning the measurable parameters would change too much when the one-loop corrections are included but, of course, the precise number of events will do.

Later we will also study the effect of the running of the parameters on our results which in some way takes into account part of the one-loop contribution, but, in any case, our approach is the simplest one including the right dependence on the chiral lagrangian parameters.

Now we describe how we have calculated the cross sections of the above mentioned processes. From the quantized chiral Lagrangian that appears in eq.(7) we obtain the corresponding Feynman rules (see Appendix). Then we compute, at tree level, the amplitudes for the different subprocesses contributing to the $Z^{0} Z^{0}$ or $W^{ \pm} Z^{0}$ final state, using a REDUCE code, and then the corresponding cross-sections. We calculate them in the center of mass frame, with a total energy $\sqrt{\hat{s}}$. There, the four momenta of the interacting particles are:

$$
\begin{aligned}
& p_{1}=\left(E_{1}, 0,0, p\right) \\
& p_{2}=\left(E_{2}, 0,0, p\right) \\
& p_{3}=\left(E_{3}, p^{\prime} \sin \theta, 0, p^{\prime} \cos \theta\right) \\
& p_{4}=\left(E_{4},-p^{\prime} \sin \theta, 0,-p^{\prime} \cos \theta\right)
\end{aligned}
$$

Here, $E_{i}$ are the particle energies, and $p$ and $p^{\prime}$ are, respectively, the magnitudes of the three-momenta of the initial and the final states.

$$
\begin{aligned}
p & =\frac{1}{2 \sqrt{\hat{s}}}\left[\hat{s}^{2}-2 \hat{s}\left(m_{1}^{2}+m_{2}^{2}\right)+\left(m_{2}^{2}-m_{1}^{2}\right)^{2}\right]^{1 / 2} \\
p^{\prime} & =\frac{1}{2 \sqrt{\hat{s}}}\left[\hat{s}^{2}-2 \hat{s}\left(m_{3}^{2}+m_{4}^{2}\right)+\left(m_{4}^{2}-m_{3}^{2}\right)^{2}\right]^{1 / 2} \\
E_{i} & =\sqrt{m_{i}^{2}+p_{i}^{2}} \quad i=1, . .4 \\
\hat{s} & =\left(p_{1}+p_{2}\right)^{2} \\
\hat{t} & =\left(p_{1}-p_{3}\right)^{2} \\
\hat{u} & =\left(p_{1}-p_{4}\right)^{2}
\end{aligned}
$$

where $\hat{s}, \hat{t}$ and $\hat{u}$ are the Mandelstam variables. The differential cross section is given by this expression:

$$
d \hat{\sigma}=\frac{1}{32 \pi \hat{s}} \frac{p^{\prime}}{p} \sum|M|^{2} d \cos \theta
$$


where $M$ is the helicity amplitude, and the $\Sigma$ symbol refers to the sum of all the final polarizations and the average on the initial ones. In the case of electroweak gauge bosons scattering, where we separate the transversal and longitudinal polarizations in the initial state (as we will see in section 5), we take into account the different helicities contributing to $T T, T L, L T$ and $L L$ polarizations of the initial bosons, to do this average. We also include the corresponding branching ratios to the gold-plated events.

Let us consider now the processes corresponding to the elastic collision of gauge bosons (fusion processes). Symbolically this reaction can be written as:

$$
V_{1}\left(p_{1}, \lambda_{1}\right) V_{2}\left(p_{2}, \lambda_{2}\right) \rightarrow V_{3}\left(p_{3}, \lambda_{3}\right) V_{4}\left(p_{4}, \lambda_{4}\right)
$$

We choose the following polarization vectors in the helicity base $\left(\lambda_{i}= \pm 1,0\right)$ :

$$
\begin{aligned}
\epsilon_{1}^{ \pm} & =\frac{1}{\sqrt{2}}(0, \mp 1,-\mathrm{i}, 0) \\
\epsilon_{1}^{0} & =\frac{1}{m_{V_{1}}}\left(p, 0,0, E_{1}\right) \\
\epsilon_{2}^{ \pm} & =\frac{1}{\sqrt{2}}(0, \mp 1, \mathrm{i}, 0) \\
\epsilon_{2}^{0} & =\frac{1}{m_{V_{2}}}\left(p, 0,0,-E_{2}\right) \\
\epsilon_{3}^{ \pm} & =\frac{1}{\sqrt{2}}(0, \mp \cos \theta,-\mathrm{i}, \pm \sin \theta) \\
\epsilon_{3}^{0} & =\frac{1}{m_{V_{3}}}\left(p^{\prime}, E_{3} \sin \theta, 0, E_{3} \cos \theta\right) \\
\epsilon_{4}^{ \pm} & =\frac{1}{\sqrt{2}}(0, \mp \cos \theta, \mathrm{i}, \pm \sin \theta) \\
\epsilon_{4}^{0} & =\frac{1}{m_{V_{4}}}\left(p^{\prime},-E_{4} \sin \theta, 0,-E_{4} \cos \theta\right)
\end{aligned}
$$

The number of independent helicity amplitudes in fusion processes of massive gauge bosons, such as $Z^{0} Z^{0} \rightarrow Z^{0} Z^{0}, W^{+} W^{-} \rightarrow Z^{0} Z^{0}, W^{+} Z^{0} \rightarrow W^{+} Z^{0}$ or $W^{-} Z^{0} \rightarrow W^{-} Z^{0}$, is $3^{4}=81$. The photon has only two polarized transverse states and therefore, in the processes $W^{+} \gamma \rightarrow W^{+} Z^{0}$ or $W^{-} \gamma \rightarrow W^{-} Z^{0}$ the number of helicity amplitudes is $2 \times$ $3^{3}=54$. As our chiral Lagrangian affecting the boson scattering is invariant under $C, P$ and $T$ transformations, we can derive many relations between different helicity amplitudes. However we have not used those relations to save computations. Instead we have calculated all the helicity amplitudes in each process and the relations between them have been only used to check our results. 


\section{$3.1 \quad Z^{0} Z^{0}$ final state}

Now we concentrate in the different processes that contribute to the final state $Z^{0} Z^{0}$ [8]:

$$
\begin{aligned}
g g & \rightarrow Z^{0} Z^{0} \\
q \bar{q} & \rightarrow Z^{0} Z^{0} \\
Z_{T}^{0} Z_{T}^{0} & \rightarrow Z^{0} Z^{0} \\
Z_{T}^{0} Z_{L}^{0} & \rightarrow Z^{0} Z^{0} \\
Z_{L}^{0} Z_{T}^{0} & \rightarrow Z^{0} Z^{0} \\
Z_{L}^{0} Z_{L}^{0} & \rightarrow Z^{0} Z^{0} \\
W_{T}^{+} W_{T}^{-} & \rightarrow Z^{0} Z^{0} \\
W_{T}^{+} W_{L}^{-} & \rightarrow Z^{0} Z^{0} \\
W_{L}^{+} W_{T}^{-} & \rightarrow Z^{0} Z^{0} \\
W_{L}^{+} W_{L}^{-} & \rightarrow Z^{0} Z^{0}
\end{aligned}
$$

Note that we separate the contribution of the different polarizations channels in the initial state, since different polarizations will have different luminosities in $p p$ collisions. In particular we use the Weizsaker-Williams [15] and the effective $W$ approximation [16] to compute the $V_{1} V_{2}$ pair luminosity in the $L H C$ beams. Therefore, we have to divide these cross-sections into the contributions coming from the different gauge boson initial polarizations.

On the other hand, it is well known that at supercollider energies the one-loop process $g g \rightarrow Z Z$ is not negligible. The gluon-gluon fusion cross section was calculated in the Minimal Standard Model, $M S M$ (with just one Higgs doublet) via one-loop of quarks by Glover and Van deer Bij [17]. The corresponding diagrams are shown in Figure 1 . As we are using a chiral Lagrangian description of the ESB sector we do not include the contribution coming from diagrams with the Higgs boson in our computations.

The quark-antiquark annihilation represents the main source of $Z^{0} Z^{0}$ pairs in $p p$ colliders like the $L H C$. As in the case of gluon fusion, there is no dependence on the chiral parameters in the calculation, at our level of approximation. The cross section at tree level, which only receives contribution from the $t$ and $u$ channels (Figure 5), has the same well-known expression as in the $M S M$.

The $Z^{0} Z^{0}$ fusion calculated using the chiral Lagrangian, only receives contribution from the vertex $Z^{0} Z^{0} Z^{0} Z^{0}$ (Figures 3 and 6). The only dependence on the effective Lagrangian parameters is through the following combination eq.(32):

$$
\alpha_{4}+\alpha_{5}+2\left(\alpha_{6}+\alpha_{7}+\alpha_{10}\right)
$$


Again we can use the relations derived from the $C, P$ and $T$ to relate the helicity amplitudes and thus to check our results. These relations are:

$$
\begin{array}{lll}
\left|M_{\lambda_{1} \lambda_{2} \lambda_{3} \lambda_{4}}\right| & \stackrel{C}{=}\left|M_{\lambda_{2} \lambda_{1} \lambda_{4} \lambda_{3}}\right| \\
\left|M_{\lambda_{1} \lambda_{2} \lambda_{3} \lambda_{4}}\right| & \stackrel{P}{=} & \left|M_{-\lambda_{1}-\lambda_{2}-\lambda_{3}-\lambda_{4}}\right| \\
\left|M_{\lambda_{1} \lambda_{2} \lambda_{3} \lambda_{4}}\right| & \stackrel{T}{=} & \left|M_{\lambda_{3} \lambda_{4} \lambda_{1} \lambda_{2}}\right|
\end{array}
$$

In order to relate some helicity amplitudes we have also taken into account whether the scattering particles are identical or not., There are still 15 independent amplitudes remaining after applying these symmetry relations.

The $W^{+} W^{-} \rightarrow Z^{0} Z^{0}$ reaction represents another source of $Z^{0} Z^{0}$ pairs from $p p$ beams. Every helicity amplitude in this process receives contributions from the $t$ and $u$ channels, by exchanging a $W$ or a $\pi$, and directly from the vertex $W^{+} W^{-} Z^{0} Z^{0}$ (Figure 7 ). Therefore we can write:

$$
M_{\lambda_{1} \lambda_{2} \lambda_{3} \lambda_{4}}=\left[M_{t_{1}}+M_{t_{2}}+M_{u_{1}}+M_{u_{2}}+M_{4}\right]_{\lambda_{1} \lambda_{2} \lambda_{3} \lambda_{4}}
$$

where the subscript 1(2) is referred to the exchange of a gauge boson (Goldstone boson) through the corresponding channel. There are 81 helicity amplitudes, but the number of independent $M_{\lambda_{1} \lambda_{2} \lambda_{3} \lambda_{4}}$ is reduced to 25 by means of symmetry relations derived from $P$ and $C$ invariance.

As we expected, the amplitudes that we have obtained satisfy these equalities. On the other hand, all the chiral parameters, $\alpha_{k}$ (but $\alpha_{10}$ ), affect this process as we can deduce from the Feynman rules eqs.(31 and 32) and from the different diagrams that contribute to the collision $W^{+} W^{-} \rightarrow Z^{0} Z^{0}$.

\section{$3.2 \quad W^{ \pm} Z^{0}$ final state}

As it was previously said, we have also studied the $W^{ \pm} Z^{0}$ final state. The different sources for $W^{ \pm} Z^{0}$ pairs in $p p$ colliders at tree level order are the following:

$$
\begin{aligned}
q \bar{q}^{\prime} & \rightarrow W^{+} Z^{0} \\
q^{\prime} \bar{q} & \rightarrow W^{-} Z^{0} \\
W_{T}^{+} Z_{T}^{0} & \rightarrow W^{+} Z^{0} \\
W_{T}^{+} Z_{L}^{0} & \rightarrow W^{+} Z^{0} \\
W_{L}^{+} Z_{T}^{0} & \rightarrow W^{+} Z^{0} \\
W_{L}^{+} Z_{L}^{0} & \rightarrow W^{+} Z^{0}
\end{aligned}
$$




$$
\begin{aligned}
W_{T}^{-} Z_{T}^{0} & \rightarrow W^{-} Z^{0} \\
W_{T}^{-} Z_{L}^{0} & \rightarrow W^{-} Z^{0} \\
W_{L}^{-} Z_{T}^{0} & \rightarrow W^{-} Z^{0} \\
W_{L}^{-} Z_{L}^{0} & \rightarrow W^{-} Z^{0} \\
W_{T}^{+} \gamma & \rightarrow W^{+} Z^{0} \\
W_{L}^{+} \gamma & \rightarrow W^{+} Z^{0} \\
W_{T}^{-} \gamma & \rightarrow W^{-} Z^{0} \\
W_{L}^{-} \gamma & \rightarrow W^{-} Z^{0}
\end{aligned}
$$

where we call $q=u, c$ and $q^{\prime}=d, s, b$.

Most of the boson pairs $W^{ \pm} Z^{0}$ are obtained in $p p$ colliders via quark-antiquark annihilations. Using the chiral Lagrangian framework we calculate the corresponding cross section at tree level order. As we can see in Figure 8 , three standard $s, t$ and $u$ diagrams contribute, but the new physics coming from the non-linear lagrangian is isolated in the three boson vertex, which in this process affects only the $s$ channel.

$$
M=M_{s}+M_{t}+M_{u}
$$

If we look at the Feynman rules (Figure 2) we can deduce that the set of $\alpha_{k}$ parameters contributing to this process are $\alpha_{1}, \alpha_{2}, \alpha_{3}, \alpha_{8}, \alpha_{9}, \alpha_{11}, \alpha_{12}$ and $\alpha_{13}$ eq.(31). In the hadronic collider case, the three boson vertex has been studied in this process with chiral Lagrangians, in [18] at tree level, using the $E T$ (by J.Bagger, et.al. in [8]), or in our previous study 19 for the case $g^{\prime}=0$ and including the running of the couplings. In this last case we analyzed the sensitivity to the $\alpha_{3}$ parameter. Here we work firstly at tree level order, but including the dependence on the $\alpha_{k}$ parameters in all the channels to produce $W^{ \pm} Z^{0}$ pairs in $p p$ colliders. Finally we will include the running parameters effect in section 8 .

Other mechanism to obtain $W^{ \pm} Z^{0}$ pairs is through $W^{ \pm} Z^{0}$ or $W^{ \pm} \gamma$ collisions transversally or longitudinally polarized at the initial state. In both cases the different polarization amplitudes are obtained by adding the contribution of the $s$ and $u$ channels and the four gauge boson vertex in (Figures 9 and 10). The exchanged particles in the $s$ and $u$ diagrams are a gauge boson $\left(s_{1}, u_{1}\right)$ and a $G B\left(s_{2}, u_{2}\right)$, respectively.

$$
M_{\lambda_{1} \lambda_{2} \lambda_{3} \lambda_{4}}=\left[M_{s_{1}}+M_{s_{2}}+M_{u_{1}}+M_{u_{2}}+M_{4}\right]_{\lambda_{1} \lambda_{2} \lambda_{3} \lambda_{4}}
$$

In the $W^{ \pm} Z^{0} \rightarrow W^{ \pm} Z^{0}$ collisions there are initially $2 \times 3^{4}$ helicity amplitudes. This 2 factor can be dropped using $C$ invariance that relates the magnitude of the helicity amplitudes $M_{\lambda_{1} \lambda_{2} \lambda_{3} \lambda_{4}}$ in the process with $W^{+}$and with $W^{-}$. If we apply the relations between amplitudes derived from $P$ and $T$ invariance, there will be only 25 remaining 
amplitudes. These subprocesses cross sections are affected by all the $\alpha_{k}$, but $\alpha_{10}$, as we can deduce from the Feynman rules eqs. 31 and 32).

The number of helicity amplitudes corresponding to the processes $W^{ \pm} \gamma \rightarrow W^{ \pm} Z^{0}$ is $2 \times\left(2 \times 3^{3}\right)$. The 2 factor disappears when we take into account $C$ invariance as in the previous case. The number of remaining amplitudes is reduced again, by another 2 factor after applying $P$ invariance. Therefore in these processes we have $3^{3}=27$ independent helicity amplitudes that we calculate using the Feynman rules shown in eqs.(31 and 32). Here we can observe that the set of the chiral lagrangian parameters affecting the $W^{ \pm} \gamma \rightarrow W^{ \pm} Z^{0}$ cross sections is the following: $\alpha_{0}, \alpha_{1}, \alpha_{2}, \alpha_{3}, \alpha_{8}, \alpha_{9}, \alpha_{11}$, $\alpha_{12}$ and $\alpha_{13}$.

In order to see the relative importance of the different channels we have evaluated the contribution to the total cross section to obtain $Z^{0} Z^{0}$ and $W^{ \pm} Z^{0}$ pairs in $p p$ colliders from the different considered subprocesses in a typical example. We have chosen the $\alpha_{k}$ parameters that mimic the $M S M$ with a heavy scalar Higgs whose mass is $m_{H}=1 T e V$. The rates for the contributions of the different subprocesses to the total $Z^{0} Z^{0}$ final state, $q \bar{q}, g g, Z^{0} Z^{0}$ and $W^{+} W^{-}$are, respectively: $45,17,3,35 \%$ and to the total $W^{ \pm} Z^{0}$ the rates are $61,22,17 \%$ coming from $q \bar{q}^{\prime}, W^{ \pm} Z^{0}$ and $W^{ \pm} \gamma$ fusion.

As it has been discussed above, the essential point in our calculations is that we have not used the ET [9, 10] to obtain the scattering amplitudes of the gauge bosons as it is customary. We have calculated the corresponding tree level amplitudes for the gauge bosons and projected them into their transversal or longitudinal components. Therefore we do not have the limitations that appear when the ET and the chiral lagrangian formulation of the $E S B$ are used together 91. The chiral lagrangian approach provides a low-energy description of the $G B$ dynamics as an expansion on the momenta over $4 \pi v$ and the $E T$ refers to the large energy relation between $G B$ and the longitudinally polarized gauge bosons $S$ matrix elements. In our case we only need to fix an upper energy bound (as it was said $E_{\max }=1.5 T e V$ ) so that we can safely apply $\chi P T$.

However, the ET can be used as a helpful tool to test our calculated longitudinally polarized amplitudes. In order to check our results we have compared our tree level expressions with those calculated applying the ET to the corresponding Goldstone boson amplitudes. We have found both amplitudes to agree at lowest order in $g$ and $g^{\prime}$ for the cases $Z_{L}^{0} Z_{L}^{0} \rightarrow Z_{L}^{0} Z_{L}^{0}, W_{L}^{+} W_{L}^{-} \rightarrow Z_{L}^{0} Z_{L}^{0}$ and $W_{L}^{ \pm} Z_{L}^{0} \rightarrow W_{L}^{ \pm} Z_{L}^{0}$ as it was expected according to the results of [10. However, the details of this comparison will be described in detail elsewhere [21] since they concern the applicability of the ET which has not been used in the present computation. Here we only wanted to quote that our results are compatible with the information that one could have obtained from the $E T$ on the above mentioned processes. 


\section{Signatures and background}

As it was stressed in the previous section we have calculated the tree level scattering amplitudes, up to order $p^{4}$, corresponding to all the helicity states of the gauge bosons, without using the ET. This fact allows us to include the dependence on the $\alpha_{k}$ parameters in all the helicity amplitudes. In contrast, when the ET is used, the only channel where the $\alpha_{k}$ coefficients are taken into account is the following:

$$
V_{L} V_{L} \rightarrow V_{L} V_{L} \quad\left(V=Z^{0}, W^{ \pm}\right)
$$

The definition of this channel as the signal was considered appropriate because the $V_{L} V_{L} \rightarrow V_{L} V_{L}$ channels are expected to be strongly interacting at high energies, if the $G B$ are, due to the ET. However, our computation makes it possible for the first time to study the effect of a strongly interacting ESB in other gauge boson polarization states by means of the $\alpha_{k}$ parameters. In fact, the aim of this work is to see how measurable will these parameters be at the $L H C$. In order to make this point more precise we must define the statistical significance corresponding to some given value of the chiral parameters $\alpha_{k}$. With this goal in mind we define our signal and our background as:

$$
\begin{gathered}
\text { (Signal) } \quad n_{S}=\left|N(\{\alpha\})-N\left(\left\{\alpha^{0}\right\}\right)\right| \\
\text { (Background) } n_{B}=N\left(\left\{\alpha^{0}\right\}\right)
\end{gathered}
$$

where $N\{\alpha\}, N\left\{\alpha^{0}\right\}$ are the total number of $Z^{0} Z^{0}$ or $W^{ \pm} Z^{0}$ pairs obtained for some given experimental cuts when the chiral parameters have been set to the values $\{\alpha\}$ or $\left\{\alpha^{0}\right\}$. The background is defined in terms of some reference model $\left\{\alpha^{0}\right\}$. For simplicity this model has been taken as the one with all the parameters set to zero i.e. $\alpha_{k}^{0}=0$. We call it Zero Model and, incidentally, it corresponds to the $M S M$ with an infinite Higgs mass.

For the final state $Z^{0} Z^{0}$ we have considered the following processes that contribute to the signal and the background:

$$
\begin{aligned}
Z^{0} Z^{0} & \rightarrow Z^{0} Z^{0} \\
W^{+} W^{-} & \rightarrow Z^{0} Z^{0}
\end{aligned}
$$

In addition we include the $Z^{0} Z^{0}$ production via gluon fusion and $q \bar{q}$ annihilation which do not depend on $\alpha_{k}$ and therefore contribute only to the background. The experimental signature for this process consists of four leptons as the result of the $Z^{0} Z^{0}$ decays. This 
gives a clean and distinct signal because the $Z^{0} Z^{0}$ pairs can be fully reconstructed. The disadvantage is the rather small leptonic branching ratio $(0.44 \%)$.

In the $W^{ \pm} Z^{0}$ case we have considered the channels:

$$
\begin{aligned}
W^{ \pm} Z^{0} & \rightarrow W^{ \pm} Z^{0} \\
W^{ \pm} \gamma & \rightarrow W^{ \pm} Z^{0} \\
q \overline{q^{\prime}} & \rightarrow W^{ \pm} Z^{0}
\end{aligned}
$$

We focus our attention on the gold-plated events where the $W^{ \pm}$and $Z^{0}$ decay to the charged leptonic final states $(l=e, \mu, \nu)$. The corresponding branching ratio is $1.3 \%$. All these cross sections depend on the chiral $\alpha_{k}$ parameters so that they are taken into account in the signature and background calculations.

The main source of $Z^{0} Z^{0}$ or $W^{ \pm} Z^{0}$ pairs in $p p$ colliders is via quark-antiquark annihilation. The total production rate of $g g \rightarrow Z^{0} Z^{0}$, in the studied cases, is $20-50 \%$ than that from $q \bar{q} \rightarrow Z^{0} Z^{0}$ depending on the top quark mass (we have chosen $m_{t}=170 \mathrm{GeV}$ ). On the other hand, the production rates of $Z^{0} Z^{0}$ or $W^{ \pm} Z^{0}$, via gauge boson fusion are suppressed by powers of $\left(\alpha / \sin ^{2} \theta_{w}\right)$ due to the application of the $W$ effective approximation [15, 16] to obtain the initial bosons from $p p$ beams, as we will see in next section.

\section{The proton and gauge boson structure functions}

Here we describe how to compute the total cross sections of the different processes studied to obtain $Z^{0} Z^{0}$ or $W^{ \pm} Z^{0}$ pairs in $p p$ colliders.

$$
\begin{aligned}
& p p \rightarrow\left(q \bar{q} \rightarrow V_{3} V_{4}\right)+X \\
& p p \rightarrow\left(g g \rightarrow Z^{0} Z^{0}\right)+X \\
& p p \rightarrow\left(V_{1} V_{2} \rightarrow V_{3} V_{4}\right)+X
\end{aligned}
$$

We have to integrate the differential cross section for the subprocess, $(d \sigma / d \cos \theta)$, with the distribution functions of the quark, antiquark and gluon (given by $f_{i}, f_{j}$ and $g$ ) inside the proton:

$$
\begin{aligned}
\sigma\left(p p \rightarrow\left(q \overline{q^{\prime}} \rightarrow V_{3} V_{4}\right)+X\right)= & \sum_{i, j} \iiint d x_{1} d x_{2} d \cos \theta f_{i}\left(x_{1}, Q^{2}\right) f_{j}\left(x_{2}, Q^{2}\right) \\
& \frac{d \hat{\sigma}}{d \cos \theta}\left(q \overline{q^{\prime}} \rightarrow V_{3} V_{4}\right)
\end{aligned}
$$




$$
\begin{gathered}
\sigma\left(p p \rightarrow\left(g g \rightarrow Z^{0} Z^{0}\right)+X\right)=\iiint_{d \hat{\sigma}} d x_{1} d x_{2} d \cos \theta g\left(x_{1}, Q^{2}\right) g\left(x_{2}, Q^{2}\right) \\
\left.\frac{d \cos \theta}{d g} \rightarrow Z^{0} Z^{0}\right)
\end{gathered}
$$

These formulae are used to compute the processes:

$$
\begin{aligned}
q \bar{q} & \rightarrow Z^{0} Z^{0} \\
\bar{q} \bar{q}^{\prime} & \rightarrow W^{ \pm} Z^{0} \\
g g & \rightarrow Z^{0} Z^{0}
\end{aligned}
$$

In order to compute the number of events of $Z^{0} Z^{0}$ and $W^{ \pm} Z^{0}$ produced in $p p$ collisions via gauge boson fusion we apply the effective $W$ approximation [15, [16] and we use the formula:

$$
\begin{aligned}
\sigma\left(p p \rightarrow\left(V_{1} V_{2} \rightarrow V_{3} V_{4}\right)+X\right)= & \sum_{i, j} \iint d x_{1} d x_{2} d \cos \theta f_{i}\left(x_{1}, Q^{2}\right) f_{j}\left(x_{2}, Q^{2}\right) \\
& \iint d \hat{\tau} d \hat{\eta} \frac{\partial^{2} L}{\partial \hat{\tau} \partial \hat{\eta}} \frac{d \hat{\sigma}}{d \cos \theta}\left(V_{1} V_{2} \rightarrow V_{3} V_{4}\right)
\end{aligned}
$$

Thus the total cross section in $p p$ colliders (like $L H C$ ) can be written as the result of the convolution of the subprocess cross section with the $V_{1} V_{2}$ pair luminosity in $p p$ beams. This luminosity is calculated from the convolution of the double bremsstrahlung of the $\left(V_{1} V_{2}\right)$ from the quark structure functions. Thus, $\partial^{2} L / \partial \hat{\tau} \partial \hat{\eta}$ is the luminosity function for the gauge boson pair $V_{1}^{h} V_{2}^{h}$ to be radiated from the quark pair $q_{i} q_{j}$. It depends on the helicity state, transversal or longitudinal, of the initial bosons $V_{1}$ and $V_{2}$. Therefore we have to separate in our computations the contribution of the different polarization channels of the initial bosons.

$$
\begin{aligned}
Z^{0} Z^{0} & \rightarrow Z^{0} Z^{0} \\
W^{+} W^{-} & \rightarrow Z^{0} Z^{0} \\
W^{ \pm} Z^{0} & \rightarrow W^{ \pm} Z^{0} \\
W^{ \pm} \gamma & \rightarrow W^{ \pm} Z^{0}
\end{aligned}
$$

The amplitudes and differential cross sections for all these processes have been obtained as it was described in the previous sections. According to the effective $W$ approach we take the following functions corresponding to the probability of a gauge boson $V_{T}$ or $V_{L}$ 
$\left(V=W^{ \pm}, Z^{0}\right)$ or a photon, to be radiated from the quark $q$, with a momentum fraction of the quark, $x$.

$$
\begin{aligned}
f_{q / V^{T}}(x) & =f_{V} \frac{x^{2}+2(1-x)}{2 x} \ln \left(\frac{E^{2}}{M_{V}^{2}}\right) \quad V=W, Z \\
f_{q / V^{L}}(x) & =f_{V} \frac{1-x}{x} \\
f_{q / \gamma}(x) & =\frac{\alpha}{2 \pi} \epsilon_{q}^{2} \frac{1+(1-x)^{2}}{x} \ln \left(\frac{E^{2}}{m_{q}^{2}}\right)
\end{aligned}
$$

where the values of the $f_{V_{i}^{\prime} s}$ depend on the particular gauge boson as well as on the type of quark that it comes from

$$
\begin{aligned}
f_{W}(x) & =\frac{\alpha}{4 \pi x_{W}} \\
f_{Z_{u \bar{u}}}(x) & =\frac{\alpha}{16 \pi x_{W}\left(1-x_{W}\right)}\left[1+\left(1-\frac{8}{3} x_{W}\right)^{2}\right] \\
f_{Z_{d \bar{d}}}(x) & =\frac{\alpha}{16 \pi x_{W}\left(1-x_{W}\right)}\left[1+\left(1-\frac{4}{3} x_{W}\right)^{2}\right]
\end{aligned}
$$

where $x_{W}=\sin ^{2} \theta_{W}$.

The variables $\tau$ and $\eta$ are related to the momentum fractions of the quarks by $x_{1,2}=\sqrt{\tau} e^{ \pm \eta}$. The connection between the variables $\hat{\tau}, \hat{\eta}$ and the momentum fractions of $V_{1}, V_{2}$ respect to $q_{i}, q_{j}, \hat{x_{1}}$ and $\hat{x_{2}}$ is given by $\hat{x_{1,2}}=\sqrt{\hat{\tau}} e^{ \pm \hat{\eta}}$. Note that the rate of transversally polarized gauge bosons obtained is enhanced by the logarithmic factors eq.(14) with respect to the longitudinal gauge boson production.

The structure functions of quarks and gluons we use are those of $E H L Q$ [22], set $I I$ with $\Lambda=290 \mathrm{MeV}$ and we neglect the contribution of the top quark to the proton sea. However we have also studied the effect of changing the structure function on our results (see below). The assignment for the $Q^{2}$ appearing in the distribution functions is $Q^{2}=\hat{s}$ for $q \overline{q^{\prime}}$ and $g g$ processes, and $Q^{2}=M_{W}^{2}$ for elastic gauge bosons scattering processes. The resulting integrals are computed using the VEGAS Monte Carlo program [23].

With the described machinery we have built up a big code which computes the total number of expected gold-plated events (for some given $L H C$ integrated luminosity) in terms of the chiral parameters $\alpha_{k}$ and the final state cuts .

Initially we impose the following cuts on the invariant mass of the weak boson pair, the transverse momentum of the final $Z^{0}$ boson and the rapidities of both bosons: 


$$
\begin{aligned}
& 200 \mathrm{GeV} \leq \sqrt{\hat{s}} \leq 1500 \mathrm{GeV} \\
& p_{T Z} \geq 10 \mathrm{GeV} \\
& \left|y_{1_{\max }}\right| \leq\left|y_{2_{\max }}\right|=2.5
\end{aligned}
$$

From now on, we will call them the minimal cuts. The upper limit in the invariant mass ensures the validity of $\chi P T$ approach. For the rest of the paper we compute the number of $Z^{0} Z^{0}$ and $W^{ \pm} Z^{0}$ obtained in the $L H C$ with an integrated luminosity of $3 \times 10^{5} \mathrm{pb}^{-1}$. This approximately corresponds to a total $L H C$ working time of 1 year $\left(3 \times 10^{7} \mathrm{sec}\right)$ assuming a luminosity $\mathcal{L}=10^{34} \mathrm{~cm}^{-2} \mathrm{~s}^{-1}$.

\section{Parameter sensitivity, optimal cuts and measur- able parameters}

In previous sections we have presented in detail how we have computed the number of $Z^{0} Z^{0}$ or $W^{ \pm} Z^{0}$ pairs obtained at the $L H C$. If we fix the integrated luminosity, the $p p$ center of mass frame energy for the $L H C(\sqrt{s}=16 \mathrm{TeV})$, and the upper bound on the invariant mass at the subprocesses $\left(\sqrt{\hat{s}_{\max }}=1.5 \mathrm{TeV}\right)$, the total cross sections will depend on the chiral $\alpha_{k}$ parameters and on some kinematical cuts. For the sake of simplicity we will only study the subset of the $\alpha_{k}$ coefficients which is needed to reabsorb the one-loop divergences obtained calculating with the chiral Lagrangian to lowest order. They are $\alpha_{0}$, $\alpha_{1}, \alpha_{2}, \alpha_{3}, \alpha_{4}$ and $\alpha_{5}$ [13] (however, our code includes as well the contribution of the other chiral parameters). There is also the possibility to modify our results choosing different kinematical cuts, $c_{l}\left(c_{1}=\sqrt{\hat{s}_{\min }}, c_{2}=p_{T Z_{\min }}\right.$ and $\left.c_{3}=y_{1_{\max }}=y_{2_{\max }}\right)$. Thus we can define two vectors: $\alpha$ and $c$, as follows:

$$
\begin{aligned}
\alpha & =\left(\alpha_{0}, \alpha_{1}, \alpha_{2}, \alpha_{3}, \alpha_{4}, \alpha_{5}\right) \\
c & =\left(c_{1}, c_{2}, c_{3}\right)
\end{aligned}
$$

which are inputs of our code; whereas the number of $Z^{0} Z^{0}(i=1)$ or $W^{ \pm} Z^{0}(i=2)$ events is the output. Therefore we write the result of our computations as $N^{(i)}(\alpha ; c)$. In principle one could expand these functions around the Zero Model (we defined it previously

as $\left.\left\{\alpha_{k}^{0}=0, \forall k\right\}\right)$ and the minimal cuts defined above so that one could write:

$$
\begin{aligned}
N^{(i)}(\alpha ; c)= & N^{(i)}\left(\alpha^{0} ; c^{\min }\right)+\left.\sum_{k=0}^{5} \frac{\partial N^{(i)}}{\partial \alpha_{k}}\right|_{\alpha_{k}=0} \alpha_{k}+\left.\sum_{l=1}^{3} \frac{\partial N^{(i)}}{\partial c_{l}}\right|_{c_{l}=c_{l}^{\min }}\left(c_{l}-c_{l}^{\min }\right) \\
& +O\left(\alpha^{2}\right)+O\left(\left(c-c^{\min }\right)^{2}\right)
\end{aligned}
$$


If the dependence of the number of events on the parameters and the cuts were approximately linear, this formula could be used to compute $N^{(i)}(\alpha ; c)$. However, we will see that this is not always the case. Indeed, $N^{(i)}(\alpha ; c)$ is a polynomial in $\alpha_{k}$ and nonlinear terms can become important even for moderate values of these parameters.

In order to see how the number of events changes when one of the $\alpha_{k}$ parameters varies by some amount $\Delta \alpha_{k}$, we fix the kinematical bounds $c_{l}$ to the minimal cuts and set the other chiral parameters to zero. We choose the following set of values for $\Delta \alpha_{k}$ :

$$
\Delta \alpha_{k}= \pm 10^{-3}, \pm 5 \times 10^{-3}, \pm 10^{-2}
$$

The result of our computations can be found in Figures 11, to 16. We can also define the sensitivity function $s_{k}^{(i)}(c)$ associated to the $\alpha_{k}$ parameter, with the kinematical cuts $c$, and for the final state $i$, as follows:

$$
\left.s_{k}^{(i)}(c) \equiv \frac{\partial N^{(i)}(\alpha ; c)}{\partial \alpha_{k}}\right|_{\alpha_{k}=0}
$$

The different sensitivity functions for the minimal cuts that we have obtained are displayed in Table 2. Obviously, the $\left|s_{k}^{(i)}(c)\right|$ values are a measure of the variations of the number of events with $\alpha_{k}$. However, they are not a direct measure of the statistical significance of the corresponding parameter variation.

We can also study the number of $Z^{0} Z^{0}$ and $W^{ \pm} Z^{0}$ event distributions with respect to the cuts given in the minimal invariant mass $\left(c_{1}\right)$, the minimal transverse momentum of $Z^{0}$ in the final state $\left(c_{2}\right)$ and in the maximal rapidity of the final bosons $\left(c_{3}\right)$. In these computations we have fixed the $\alpha$ parameters at their values in the Zero Model, and the other two kinematical cuts have been set to their minimal values. The results obtained in this way are shown in Figures 17 to 19.

In order to perform a statistical analysis, we define the $r_{k}$ function as:

$$
r_{k}^{(i)}(c)=\frac{\left|N^{(i)}\left(\alpha_{k} ; c\right)-N^{(i)}\left(\alpha^{0} ; c\right)\right|}{\sqrt{N^{(i)}\left(\alpha^{0} ; c\right)}}=\frac{n_{S}}{\sqrt{n_{B}}}
$$

The $r_{k}^{(i)}$ function is a measure of the statistical significance of the signal (corresponding to the increments $\left.\Delta \alpha_{k}\right)$ relative to the background, provided $N^{(i)}\left(\alpha_{k} ; c\right)$ is large enough to apply the Central Limit Theorem. In this case $r_{k}$ is an estimate of the number of sigmas, and therefore it defines the confidence level for the hypothesis that $\alpha_{k}$ is different from zero. In order to evaluate $r_{k}^{(i)}$ we use the number of events that was previously obtained. The resulting $r_{k}^{(i)}$ functions are shown in Figures 20 to 25.

From Figures 17 and 18, it is clear that there is no linear dependence of $N^{(i)}(\alpha ; c)$ on $c_{1}$ and $c_{2}$. Moreover, looking at Figures 11 at 16 and from Figures 20 at 25, nonlinear behaviour is observed with respect to $\alpha_{1}, \alpha_{2}$ and $\alpha_{3}$ (for $W^{ \pm} Z^{0}$ final state) nor when $\left|\alpha_{4}\right|$ or $\left|\alpha_{5}\right|$ are bigger than 0.001 in both channels, $Z^{0} Z^{0}$ and $W^{ \pm} Z^{0}$. 
By looking at Figures 20 to 25, we can see that the slope corresponding to the different curves $r_{k}^{(i)}\left(c^{\mathrm{min}}\right)$ varies according to the different values of $\alpha_{k}$ and the channel $\left(Z^{0} Z^{0}\right.$ or $\left.W^{ \pm} Z^{0}\right)$. Indeed, the greater is the slope, the higher is the statistical significance. Moreover it is already possible to observe that some of the $\alpha$ parameters have no chances to be measured at the $L H C$, at least in the way described here. For this reason, in the following we will concentrate our statistical analysis on the set of $\alpha$ parameters which can be considered as potentially measurable. Being more precise, we define a parameter $\alpha_{k}$ as potentially measurable in a given channel when the $r_{k}^{(i)}\left(c^{\mathrm{min}}\right)$ corresponding to the maximal considered variation $\left(\left|\Delta \alpha_{k}\right|=0.01\right)$, is bigger than 0.5. According to this we find that the potentially measurable $\alpha_{k}$ are the following:

- $\alpha_{3}<0$ and $\alpha_{3}>0$ (both in $Z^{0} Z^{0}$ and $W^{ \pm} Z^{0}$ channels)

- $\alpha_{4}<0\left(W^{ \pm} Z^{0}\right)$ and $\alpha_{4}>0\left(Z^{0} Z^{0}\right)$

- $\alpha_{5}<0\left(W^{ \pm} Z^{0}\right)$ and $\alpha_{5}>0\left(Z^{0} Z^{0}\right)$

In the following we carry out an statistical analysis with all these potentially measurable parameters in the corresponding final state. To do so, the next step is to look for the optimal cuts in the minimal invariant mass and transverse momentum for the detection of a certain $\Delta \alpha_{k}=\left(\alpha_{k}-\alpha_{k}^{0}\right)$ different from zero. Initially we impose the minimal cuts on the kinematical variables. The optimization procedure we use is to search for the pair of cuts $\left(\sqrt{\hat{s}}, p_{T Z}^{c}\right)$ in the kinematical region

$$
\begin{aligned}
200 \mathrm{GeV} & \leq \sqrt{\hat{s}} \leq 1500 \mathrm{GeV} \\
10 \mathrm{GeV} & \leq p_{T Z} \leq \sqrt{\hat{s}_{\max } / 4-m_{z}^{2}}
\end{aligned}
$$

that maximize the function $r_{k}^{(i)}\left(\alpha_{k} ; \sqrt{\hat{s}^{c}}, p_{T Z}^{c}\right)$ defined as follows:

$$
r_{k}^{(i)}\left(\alpha_{k} ; \sqrt{\hat{s}}^{c}, p_{T Z}^{c}\right)=\frac{\left|N^{(i)}\left(\alpha_{k} ; \sqrt{\hat{s}}>\sqrt{\hat{s}^{c}}, p_{T Z}>p_{T Z}^{c}\right)-N^{(i)}\left(\alpha_{k}^{0} ; \sqrt{\hat{s}}>\sqrt{\hat{s}^{c}}, p_{T Z}>p_{T Z}^{c}\right)\right|}{\sqrt{N^{(i)}\left(\alpha_{k}^{0} ; \sqrt{\hat{s}}>\sqrt{\hat{s}^{c}}, p_{T}>p_{T}^{c}\right)}}
$$

To find the optimal cuts for the different parameters and channels we build a bidimensional grid on the plane $\left(\sqrt{\hat{s}}, p_{T Z}\right)$ so that the different points are separated from each other by the increments $\left(\Delta \sqrt{\hat{s}}=50 \mathrm{GeV}, \Delta p_{T}=50 \mathrm{GeV}\right)$. For each pair of points in this grid $\left(\sqrt{\hat{s}^{c}}, p_{T Z}^{c}\right)$ we compute the total number of events in the Zero Model and in a ESB scenario corresponding to a certain positive or negative $\Delta \alpha_{k}$. Finally, we evaluate the $r_{k}^{(i)}\left(\alpha_{k} ; \sqrt{\hat{s}^{c}}, p_{T Z}^{c}\right)$ function. The optimal cuts, $c^{\mathrm{op}}$, are those which maximize eq. (20), and 
therefore, the confidence level. Of course, these $c^{\mathrm{op}}$ which have been found in this way depend slightly on the particular choice of $\Delta \alpha_{k}$ for each $\alpha_{k}$ parameter. In our computation we have taken the typical values:

$$
\Delta \alpha_{k}=\alpha_{k}-\alpha_{k}^{0}=\alpha_{k}= \pm 0.005
$$

The results are collected in Tables 3 and 4 . There it is displayed, for each $\Delta \alpha_{k}$ in the corresponding channel, the optimal cuts, the number of events that satisfies these cuts and the statistical significance function $r_{k}$ obtained with the minimal and the optimal cuts. In all cases we have fixed a maximal rapidity of 2.5 .

The $c^{\text {op }}$ we have obtained by maximizing the $r_{k}^{(i)}$ function eq. (20) will be considered from now on as the optimal cuts to detect either a $\Delta \alpha_{k}<0$ or a $\Delta \alpha_{k}>0$. For example, if we wanted to observe the signature corresponding to a heavy Higgs $S M$-like $E S B$ scenario, the best choice would be the $Z^{0} Z^{0}$ final state with the optimal cuts obtained for $\Delta \alpha_{5}>0\left(\sqrt{\hat{s}_{\min }}=1150 \mathrm{GeV}, p_{T Z \text { min }}=400 \mathrm{GeV}\right)$. This is so because, at the tree level order, the only $\alpha_{k}$ different from zero needed to mimic this scenario is $\alpha_{5}\left(\alpha_{5}=v^{2} /\left(8 m_{H}^{2}\right)\right)$.

As it can be seen in Table 4 , the optimization procedure has clearly improved the statistical significance $r_{k}$ in most of the studied cases. Only when we tried to optimize the signature corresponding to a $\alpha_{3}>0$ and $\alpha_{3}<0$, both in the $Z^{0} Z^{0}$ final state, we obtained no significant improvement with respect to the minimal cuts. Therefore we find it impossible to detect a signature of a negative or positive $\alpha_{3}$ in $Z^{0} Z^{0}$ events.

Finally, we have carried out a similar optimization procedure for $\alpha_{4}$ and $\alpha_{5}$ in which we searched for a slightly different pair of optimal cuts: $\left(\sqrt{\hat{s}_{\max }}, p_{T Z_{\text {min }}}\right)$. We obtained $c^{\mathrm{op}}=(1500 \mathrm{GeV}, 500 \mathrm{GeV})$ for $\alpha_{4}=-0.005\left(W^{ \pm} Z^{0}\right), \alpha_{4}=0.005\left(Z^{0} Z^{0}\right)$ and $\alpha_{5}=0.005$ $\left(Z^{0} Z^{0}\right)$ and the $r_{k}$ function was respectively $6.02,1.56$ and 3.82. On the other hand, we also found the optimal cuts to detect $\alpha_{5}=-0.005$ in the $W^{ \pm} Z^{0}$ final state ( $c^{\text {op }}=$ $(1500 \mathrm{GeV}, 400 \mathrm{GeV}))$ and the reached statistical significance was 2.97. These results are at most equal to those of Table 1 .

Moreover, we have repeated our whole study to find other sets of optimal cuts belonging to the parameter space, such as $\left(\sqrt{\hat{s}_{\max }}, p_{T Z_{\min }}\right),\left(\sqrt{\hat{s}_{\max }}, p_{T Z_{\max }}\right),\left(\sqrt{\hat{s}_{\min }}, p_{T Z_{\max }}\right)$. In all these cases, the statistical significances obtained are smaller than those corresponding to the first choice of $c^{\mathrm{op}}\left(c^{\mathrm{op}}=\left(\sqrt{\hat{s}_{\min }}, p_{T Z_{\text {min }}}\right)\right)$. Therefore, in the following we will apply this type of optimal cuts.

As it was mentioned in the introduction the main aim of this work is to determine which chiral Lagrangian parameters will be more easily measurable at the $L H C$ and the size of their corresponding statistical errors. Initially, we made a criterion to choose a set of potentially measurable $\alpha_{k}$ parameters, on which we have concentrated our analysis. After carrying out the described optimization procedure, we conclude that the statistical significance to measure most of these chiral Lagrangian coefficients, has clearly increased. In the following we will treat only these measurable $\alpha_{k}$. The set of the $\alpha_{k}$ parameters 
obtained as the measurable ones are contained in Tables 3 and 4 . Here we exclude the study of $\alpha_{3}$ in the $Z^{0} Z^{0}$ final state, as it was previously argued.

In order to obtain an estimation of the statistical errors $\left(\Delta \alpha_{k}\right)_{\text {stat. }}$ we will work under some hypothesis. We will assume a linear behaviour in $\alpha_{k}$ and that the number of events is large enough to consider $N^{(i)}(\alpha ; c)$ following a gaussian distribution.

At this point, we define the statistical error $\left(\Delta \alpha_{k}\right)_{\text {stat. }}$ associated to a certain $\alpha_{k}$ as the minimal value of this parameter that could be detected in the $L H C$ with a statistical significance, eq.(18), equal to one sigma, after applying its corresponding optimal cuts. Using the results displayed in Table 1 which satisfy the optimal cuts, we obtain the $\left(\Delta \alpha_{k}\right)_{\text {stat. }}$ in this way, and we show the results in Table 5. As it could be expected, the smallest $\left(\Delta \alpha_{k}\right)_{\text {stat. }}$ value corresponds to the biggest statistical significance, which is $\left(\Delta \alpha_{4}\right)_{\text {stat. }}=8.31 \times 10^{-4}$, in the $W^{ \pm} Z^{0}$ final state.

\section{The effect of the structure function indetermina- tion}

In all the previous calculations we have used the $E H L Q$ structure functions [22] (set $I I$ with $\Lambda=290 \mathrm{MeV}$ ) to obtain the total number of $Z^{0} Z^{0}$ and $W^{ \pm} Z^{0}$ events. Here we wonder how our results could be affected by the indetermination in the parton distribution functions. In order to estimate this effect we have selected two new parametrizations of the structure function: the set $M R S D-$ (Martin, Roberts and Stirling [24]) and the GRVHO (Glück, Reya and Vogt [25]). Both them exhibit a similar behaviour of sea quarks and gluon distributions at low $x$, that grow when $x \rightarrow 0$. The values of $\Lambda$ considered in these two sets are the following: $\Lambda_{\mathrm{MRS}}=230 \mathrm{MeV}, \Lambda_{\mathrm{GRV}}=200 \mathrm{MeV}$. With these new structure functions we evaluate the total number of $Z^{0} Z^{0}$ or $W^{ \pm} Z^{0}$ events, in the Zero Model with the different optimal cuts. The way we proceed is to compare our estimation of the number of standard deviations obtained by changing these parton density function sets (we will call it $r_{\text {struc. }}^{(i)}$ ), with the statistical significance corresponding to a certain signal, after having applied the optimal cuts (the $r_{k}^{(i)}\left(c^{\mathrm{op}}\right)$ functions shown in Table 4$)$. We define $r_{\text {struc. }}^{(i)}$ similarly as in eq.(18):

$$
r_{\text {struc. }}^{(i)}(c)=\frac{\left|N_{\text {set }}^{(i)}\left(\alpha^{0} ; c\right)-N_{\text {set }^{\prime}}^{(i)}\left(\alpha^{0} ; c\right)\right|}{\sqrt{N_{\text {set }}^{(i)}\left(\alpha^{0} ; c\right)}}
$$

Now, to obtain $r_{\text {struc. }}^{(i)}$ we need to evaluate the total number of events in the Zero Model, with certain kinematical cuts, $c$, but using two different parametrizations called set and set' for the structure functions. 
In Table 6 we show the results of our calculations. We represent the total number of $Z^{0} Z^{0}$ and $W^{ \pm} Z^{0}$ events obtained for the optimal cuts, when the $E H L Q$ (set II), MRSDand $G R V H O$ sets are used, as well as the number of sigmas $r_{\text {struc. }}$ eq.(22) corresponding to change one of these sets with respect to the others:

- $r_{\text {struc. }} \equiv r_{12}(E H L Q$ with respect to $M R S D-)$

- $r_{\text {struc. }} \equiv r_{13}(E H L Q$ with respect to $G R V H O)$

- $r_{\text {struc. }} \equiv r_{23}(M R S D$ - with respect to $G R V H O)$.

Now, we pay attention to the $r_{k}$ functions in Table 4 and $r_{\text {struc. }}$ in Table 6 and we compair them. We will say that the typical chosen value of $\alpha_{k}\left(\alpha_{k}=-0.005\right.$ or $\left.\alpha_{k}=0.005\right)$ can be measured in the $L H C$ with a statistical significance given by $r_{k}$, if the following inequality is verified:

$$
r_{k}>>r_{\text {struc. }}
$$

When we make $r_{\text {struc. }}=r_{12}$ or $r_{\text {struc. }}=r_{13}$, we must reject the value $\alpha_{3}=0.005$ in $Z^{0} Z^{0}$ and $W^{ \pm} Z^{0}$ channels, and $\alpha_{3}=-0.005$ in $Z^{0} Z^{0}$ final state, because relation (23) is violated. However, if we take $r_{\text {struc. }}=r_{23}$ we can measure positive and negative values of $\alpha_{3}, \alpha_{4}$ and $\alpha_{5}$ parameters in some $Z^{0} Z^{0}$ or $W^{ \pm} Z^{0}$ final state, with a statistical significance given by $r_{k}$. On the opposite, for $\alpha_{3}= \pm 0.005$ in $Z^{0} Z^{0}$ final state, eq. (23) is not fulfilled, so we can only measure these values of $\alpha_{3}$ through the $W^{ \pm} Z^{0}$ channel. This last choice $\left(r_{\text {struc. }}=r_{23}\right)$ is most appropriate since $M R S D-$ and $G R V H O$ agree much better with some recent experimental results [26] obtained at $H E R A$. In any case, it can be expected that future new experimental data coming from $H E R A$ and even from the $L H C$ itself, will allow us to reduce the size of these uncertainties $r_{\text {struc. }}$.

\section{Renormalization effects}

The other effect we are going to take into account is the dependence of the $\alpha_{k}$ parameters and the couplings $g$ and $g^{\prime}$, on the energy. Our whole previous study was carried out at tree level order. Now we want to include some quantum effects by means of the Renormalization Group Equations $(R G E)$. By that we will understand the one loop contributions coming from $\mathcal{L}^{(2)}$ and $\mathcal{L}_{\mathrm{YM}}$ plus the tree level contributions coming from $\mathcal{L}_{1}^{\prime}+\mathcal{L}^{(4)}$ to the corresponding beta functions.

As it is well known from the $R G E$, the renormalized Green function with the renormalized parameters $\lambda$ and $m$ at the renormalization scale $\mu$ and the same Green function with the parameters at another scale $\mu e^{t}$, are related by the equation: 


$$
G_{R}^{(n)}\left(p_{i}, \lambda, \mu\right)=G_{R}^{(n)}\left(p_{i}, \bar{\lambda}(t), \mu e^{t}\right) \exp \left[-n \int_{0}^{t} \gamma\left(\bar{\lambda}\left(t^{\prime}\right)\right) d t^{\prime}\right]
$$

Where we include in $\lambda$ all the $\alpha_{k}$ parameters and the electroweak coupling constants, $g$ and $g^{\prime}$. Thus the renormalized parameters are scale dependent. The $\bar{\lambda}$ stands for all the running coupling constants that depend on the renormalization scale. In order to obtain these $\bar{\lambda}$ we need to calculate the $\beta(\lambda)$ and $\gamma(\lambda)$ functions, following the well known $R G E$ techniques:

$$
\begin{aligned}
\beta(\bar{\lambda}) & =\frac{\partial \bar{\lambda}(\lambda, t)}{\partial t} \\
\gamma(\bar{\lambda}(t)) & =\frac{1}{2} \frac{\partial \log Z_{3 V}}{\partial t}
\end{aligned}
$$

At present, when using the effective theory to describe the ESB sector we only know these functions to one loop-order. The exponential factor in eq.(24) is the anomalous dimension term, that depends on the wave function renormalization. We have estimated the order of magnitude of this effect to calculate the statistical significance $r_{k}$, in a typical case, with a subprocess energy of $1 \mathrm{TeV}$. We obtained changes on $r_{k}$ of $0.1 \%$. Therefore they are completely irrelevant. Thus we neglect this contribution and we will only consider the dependence on the renormalization scale, $\mu$, of $\alpha_{k}, g$ and $g^{\prime}$, in eq.(24). We refer to this approach as the tree level approximation improved by the $R G E$.

Now, we have to calculate the running coupling constants $\alpha_{k}(\mu), g(\mu)$ and $g^{\prime}(\mu)$, and replace their tree level values in our cross section formulae by the corresponding energy dependent running coupling constants evaluated at the subprocess center of mass energy (we have taken $\mu=\sqrt{\hat{s}}$ ).

In order to do so we begin with the $\alpha_{k}$ parameters and we use the dimensional regularization scheme since it is the most appropriate for gauge theories as well as for non-linear sigma models. In 13], Longhitano obtained all the divergences that appear in one-loop diagrams calculated with the effective lagrangian at lowest order $\left(\mathcal{L}^{(2)}+\mathcal{L}_{\mathrm{YM}}\right)$. They could be absorbed by redefinitions of a subset of the $\alpha_{k}$ parameters $(k=0,1,2,3,4,5)$. By means of the dependence of $\alpha_{k}$ on the $\epsilon$ parameter [13], it is easy to obtain the following $\alpha_{k}$ running expressions:

$$
\begin{aligned}
& \alpha_{0}^{R}(\mu)=\alpha_{0}^{R}\left(\mu_{0}\right)-\frac{1}{16 \pi^{2}} \frac{3}{4} \operatorname{tg}^{2} \theta_{\mathrm{w}} \log \left(\frac{\mu_{0}}{\mu}\right) \\
& \alpha_{1}^{R}(\mu)=\alpha_{1}^{R}\left(\mu_{0}\right)-\frac{1}{16 \pi^{2}} \frac{1}{6} \operatorname{tg} \theta_{\mathrm{w}} \log \left(\frac{\mu_{0}}{\mu}\right)
\end{aligned}
$$




$$
\begin{aligned}
& \alpha_{2}^{R}(\mu)=\alpha_{2}^{R}\left(\mu_{0}\right)-\frac{1}{16 \pi^{2}} \frac{1}{12} \operatorname{tg} \theta_{\mathrm{w}} \log \left(\frac{\mu_{0}}{\mu}\right) \\
& \alpha_{3}^{R}(\mu)=\alpha_{3}^{R}\left(\mu_{0}\right)-\frac{1}{16 \pi^{2}} \frac{1}{12} \log \left(\frac{\mu_{0}}{\mu}\right) \\
& \alpha_{4}^{R}(\mu)=\alpha_{4}^{R}\left(\mu_{0}\right)+\frac{1}{16 \pi^{2}} \frac{1}{6} \log \left(\frac{\mu_{0}}{\mu}\right) \\
& \alpha_{5}^{R}(\mu)=\alpha_{5}^{R}\left(\mu_{0}\right)+\frac{1}{16 \pi^{2}} \frac{1}{12} \log \left(\frac{\mu_{0}}{\mu}\right)
\end{aligned}
$$

Apart from these equations, we need also to calculate the running of the gauge couplings $g_{R}(\mu)$ and $g_{R}^{\prime}(\mu)$. As usual we have

$$
\begin{aligned}
& g_{R}=Z_{3}^{3 / 2} Z_{1}^{-1} g_{0} \\
& g_{R}^{\prime}=Z_{3}^{\prime 1 / 2} g_{0}^{\prime}
\end{aligned}
$$

The only differences with respect to the linear model, given by $\delta Z_{3}$ and $\delta Z_{3}^{\prime}$ functions, come from the scalar sector contribution. In our case, they are

$$
\begin{aligned}
\delta Z_{3} & =\frac{g^{2}}{16 \pi^{2}} \frac{1}{6 \epsilon} \\
\delta Z_{3}^{\prime} & =\frac{g^{\prime 2}}{16 \pi^{2}} \frac{1}{6 \epsilon}
\end{aligned}
$$

Taking into account loops of scalars, fermions, ghosts and gauge bosons coming from the rest of the $S M$ particles we arrive to the following values of the $\beta$ functions eq.(25), $\beta_{g}$ and $\beta_{g^{\prime}}$ :

$$
\begin{aligned}
& \beta_{g}=-C_{g} g^{3} \\
& \beta_{g^{\prime}}=-C_{g^{\prime}} g^{\prime 3}
\end{aligned}
$$

where

$$
\begin{aligned}
C_{g} & =\frac{1}{16 \pi^{2}} \frac{13}{4} \\
C_{g^{\prime}} & =-\frac{1}{16 \pi^{2}} \frac{27}{4}
\end{aligned}
$$


The eqs.(27) are standard corresponding for gauge theories. The first one leads to asymptotic freedom since $C_{g}>0$, whereas the second corresponds to an abelian gauge theory. Therefore one has:

$$
\begin{aligned}
g_{R}^{2}(\mu) & =\frac{g_{R}^{2}\left(\mu_{0}\right)}{1+\frac{1}{16 \pi^{2}} \frac{13}{2} g_{R}^{2}\left(\mu_{0}\right) \log \frac{\mu}{\mu_{0}}} \\
g_{R}^{\prime 2}(\mu) & =\frac{g_{R}^{\prime 2}\left(\mu_{0}\right)}{1-\frac{1}{16 \pi^{2}} \frac{27}{2} g_{R}^{\prime 2}\left(\mu_{0}\right) \log \frac{\mu}{\mu_{0}}}
\end{aligned}
$$

Once we have obtained the expression giving the dependence of the $\alpha_{k}, g$ and $g^{\prime}$ on the renormalization scale eq.(28), we can study cuantitatively this effect. The way we proceed is the following:

- First we factorize the $g$ and $g^{\prime}$ coupling constants so that the cross sections appear with the same power in the electroweak couplings than in the $M S M$. We are referring only to the dominant terms. Moreover, as we can see in the Appendix, eqs.(30, 31 and 32), higher powers on these coupling constants appear as factors of the $\alpha_{k}$ parameters.

- In all our calculations, for the sake of simplicity, we are considering the physical masses [27]: $m_{W}=80.6 \mathrm{GeV}, m_{Z}=91.1 \mathrm{GeV}$ and $m_{q}=0$ (except to in $g g \rightarrow Z^{0} Z^{0}$ process where we have taken a top quark in the loop with a mass of $170 \mathrm{GeV}$ ).

- We replace in the cross section the constant values of $\alpha_{k}, g$ and $g^{\prime}$ by those which are renormalized and scale dependent, $\alpha_{k}^{R}(\mu), g_{R}(\mu)$ and $g_{R}^{\prime}(\mu)$ given by eqs. 26 and 28). We take $\mu=\sqrt{\hat{s}}$, where $\sqrt{\hat{s}}$ is the invariant mass (the center of mass energy in the considered subprocess) and $\mu_{0}=m_{Z}$. Thus we have substituted the tree level values of $\alpha_{k}, g$ and $g^{\prime}$ by the running coupling constants depending on the energy scale of the subprocess.

The assignment for the numerical values of $g_{R}\left(\mu_{0}\right), g_{R}^{\prime}\left(\mu_{0}\right)$ and $\alpha_{k}^{R}\left(\mu_{0}\right)$ parameters appearing in our formulas has been done as follows:

- The quantities $g_{R}$ and $g_{R}^{\prime}$, at the chosen scale $\mu_{0}=m_{Z}$, have been taken from recent $L E P$ data 27].

- The symmetry breaking pattern that we have considered corresponds to neglecting all the $\alpha_{k}^{R}$ parameters at the $\mu_{0}$ scale. We refer to this scenario as the Running Zero Model:

$$
\begin{array}{lll}
\alpha_{k}\left(\mu_{0}\right) & =0 & (k=0,1,2,3,4,5) \\
\alpha_{k} & =0 & (k=6,7,8,9,10,11,12,13)
\end{array}
$$


After all these changes in the program, we apply the different sets of optimal cuts previously obtained, (Table 3) and calculate the total number of events to produce $Z^{0} Z^{0}$ or $W^{ \pm} Z^{0}$. The corresponding results are displayed in Table 7 . We present for each pair of optimal cuts, the total number of $Z^{0} Z^{0}$ or $W^{ \pm} Z^{0}$ events, in the Zero Model and with $g$ and $g^{\prime}$ constant $\left(N^{(i)}\left(\alpha^{0} ; g, g^{\prime}, c\right)\right)$, and in the Running Zero Model with $g_{R}(\sqrt{\hat{s}})$ and $g_{R}^{\prime}(\sqrt{\hat{s}})\left(N^{(i)}\left(\alpha^{0}(\sqrt{\hat{s}}) ; g_{R}(\sqrt{\hat{s}}), g_{R}^{\prime}(\sqrt{\hat{s}}), c\right)\right)$. We also give an estimation of the statistical significance or the number of sigmas, $r_{\text {run. }}^{(i)}$, corresponding to include the dependence on the energy of the parameters, with respect to the tree level results. Here, we calculate $r_{\text {run. }}^{(i)}$ function analogously to eqs.(18) and (22)

$$
r_{\text {run. }}^{(i)}(c)=\frac{\left|N^{(i)}\left(\alpha^{0} ; g, g^{\prime}, c\right)-N^{(i)}\left(\alpha^{0}(\sqrt{\hat{s}}) ; g(\sqrt{\hat{s}}), g^{\prime}(\sqrt{\hat{s}}), c\right)\right|}{\sqrt{N^{(i)}\left(\alpha^{0} ; g, g^{\prime}, c\right)}}
$$

As it can be observed in Table 7, the $r_{\text {run. }}^{(i)}$ function, that reflects the relevance of this effect, is very high when we use the minimal cuts. Besides, with all the optimal cuts, the statistical significance function $r_{\text {run. }}^{(i)}$ whose values go from 1 to 4 standard deviations is also important. Thus, the effect of the running of the couplings is important and it should be taken into account if one wants to compute the total number of $Z^{0} Z^{0}$ or $W^{ \pm} Z^{0}$ events.

Now one could ask how the $r_{k}$ that were previously obtained (Table 1 ) would change if we used the improved tree level approximation with the running parameters, instead of working only at tree level order. In sight of the results contained in Table 7 and if we supposed linear behaviour we would obtain fluctuations in the statistical significance functions, $r_{k}$, varying between 2 to $15 \%$ with respect to their values calculated without including the dependence on the energy of the parameters.

We can summarize these results saying that the inclusion of the running coupling constants in our calculations leads to important variations in the total number of events, given by $r_{\text {run. }}^{(i)}$ functions. However, this effect is not so relevant when we want to determine the $r_{k}$ functions corresponding to each $\alpha_{k}$, since the differences obtained with respect to the tree level results are small. Therefore, the running of the couplings does not change significantly our previous discussion about which couplings will be measurable at the LHC.

\section{Conclusions}

Using $\chi P T$ to describe the $E S B$ sector, we have studied the different processes contributing to $Z^{0} Z^{0}$ and $W^{ \pm} Z^{0}$ final states at the $L H C$, considering only gold-plated events. The main novelty in our analysis is that we do not use the ET but we have worked explicitly with all the the gauge boson polarization states. Thus we can study the low energy region, 
after having imposed a maximal bound on the subprocess energy of $1.5 \mathrm{TeV}$. We have elaborated a FORTRAN code that generates the subprocess cross sections at tree level order, producing $Z^{0} Z^{0}$ or $W^{ \pm} Z^{0}$ pairs.

With this code we have carried out a systematical study of the possibilities for measuring the chiral parameters $\alpha_{k}$, at the $L H C$. This analysis includes an optimization procedure, that makes possible to obtain the greatest statistical significance for measuring certain values of these parameters. We have also computed their corresponding statistical errors, $\left(\Delta \alpha_{k}\right)_{\text {stat. }}$, and set the minimum values of these $\alpha_{k}$ to be unambiguously detected with a certain statistical significance at the $L H C$. The results of our studies are displayed in Tables 4 and 5. From our results it is clear that the $\alpha_{0}, \alpha_{1}$ and $\alpha_{2}$ parameters cannot be probed at the $L H C$. On the contrary, as it can be seen in Tables 1 and 5 , the $r_{k}$ function obtained for $\alpha_{4}$ and $\alpha_{5}$ can be, for some channels, greater than 3 sigmas (2 sigmas for $\left.\alpha_{3}\right)$. In fact, the parameters that could be more easily measured in the $L H C$ are $\alpha_{4}<0$ in $W^{ \pm} Z^{0}$ and $\alpha_{5}>0$ in $Z^{0} Z^{0}$ channel. As it can be expected their corresponding statistical errors are the smallest. In all our calculations, we have fixed a running time corresponding to one full $L H C$ year according to the nominal luminosity $L=10^{34} \mathrm{~cm}^{-2} \mathrm{sec}^{-1}\left(L_{\text {int. }}=3 \times 10^{5} \mathrm{pb}^{-1}\right)$. The results corresponding to different integrated luminosities can be obtained just rescaling the statistical significance by the square root of the running time.

On the other hand, we have also estimated the size of the imprecisions in our calculations due to the indetermination in the structure functions by choosing three different distribution functions. The results (which can be found in Table 6) show that the errors coming from this effect are smaller than the statistical errors obtained for the $\alpha$ parameters. Thus our present ignorance about the parton distribution functions does not restrict the measurement of the chiral parameters.

We have also tried to improve our tree level results considering the dependence on the subprocess energy of the weak couplings and the chiral parameters. The size of these effects is shown in Table 07. From our results it is clear that the total number of events changes when this effect is taken into account. However, our previous conclusions about which parameters can be probed at the $L H C$, do not change at all.

Moreover one could ask about the possibility of increasing the number of measurable chiral parameters by relaxing some of the experimental assumptions of this work. For instance we could also take into account the gauge boson hadronic decays, separate the final polarizations, etc... In that case we could certainly enhance the statistical significance and more chiral parameters could be probed. However it is not possible today to have any idea about how well this new information will be obtained at the $L H C$ and for this reason we have not considered such possibilities.

Finally we have also assumed that the $Z^{0} Z^{0}$ or $W^{ \pm} Z^{0}$ pairs produced via electroweak gauge boson fusion cannot be separated experimentally from those coming from another 
sources such as $g g$ fusion (in $Z^{0} Z^{0}$ case) or quark-antiquark annihilation. Nevertheless some forward calorimeters could, presumably, be incorporated to the $L H C$ detectors. This fact could allow for a jet tagging at a certain level of efficiency. This experimental improvement is a realistic way to increase the statistical significance functions $r_{k}$ to measure the chiral Lagrangian parameters at $L H C$. Work is in progress to treat this possibility and to analyze how much the sensibility to the $\alpha_{k}$ parameters could be enhanced.

\section{Acknowledgements}

We thank M. J. Herrero for her patient help along the more than two years that this work took to be finished, as well as D. Denegri, T. Rodrigo, J. Terrón for some interesting discussions and J. R. Peláez for reading the manuscript. We also thank support by the Ministerio de Educación y Ciencia (Spain) (CICYT AEN90-0034, AEN93-0776 and AEN93-0673). A. D. thanks to the CERN Theory Division, where the final part of this work was done, for its kind hospitality. 


\section{Appendix}

In this Appendix, we write some of the Feynman rules obtained from the quantized chiral electroweak Lagrangian $\mathcal{L}_{\mathrm{NLSM}}$ eq.(7), described in section 2.

The different contributions to $\mathcal{L}_{\mathrm{NLSM}}$ are given in eqs.(11, 2, 国 and 8), where the Landau gauge has been chosen.

We show the Feynman rules corresponding to the propagators and vertices used in our calculations:

i) The $\pi$ and $W^{ \pm}$propagators in the Landau gauge have the following expressions (see Figure 1):

$$
\begin{aligned}
-i \Delta\left(\pi^{ \pm}\right) & =\frac{i}{k^{2}} \\
i \Delta_{\mu \nu}\left(W^{ \pm}\right) & =\frac{-i}{k^{2}-m_{W}^{2}+i \epsilon}\left[g_{\mu \nu}-\frac{k_{\mu} k_{\nu}}{k^{2}}\right]
\end{aligned}
$$

ii) In the following, we write the Feynman rules corresponding to the vertices with either 3 gauge electroweak bosons $\left(\mathrm{I}_{V_{1} V_{2} V_{3}}\right)$ or to 2 gauge bosons and a $G B\left(\mathrm{I}_{V_{1} V_{2} \pi}\right)$ displayed in Figure 2 .

$$
\begin{aligned}
& \mathrm{I}_{\pi^{+} W_{\mu}^{-} Z_{\nu}^{0}}=\frac{g^{2} v}{2} \sec \theta_{W} g_{\mu \nu}\left(\sin ^{2} \theta_{W}-2 g^{2} \beta_{1}\right)+\frac{2 g^{2}}{v}\left[(q . r) g_{\mu \nu}-q_{\mu} r_{\nu}\right]\left(\alpha_{1} \sin \theta_{W}-\alpha_{8} \cos \theta_{W}+\right. \\
& \left.\alpha_{13} \sec \theta_{W}\right)+\frac{2 g^{2}}{v} \alpha_{3} \sec \theta_{W}\left[(k . r) g_{\mu \nu}-k_{\mu} r_{\nu}\right]+\frac{2 g^{2}}{v}\left[(k . q) g_{\mu \nu}-q_{\mu} k_{\nu}\right]\left(\alpha_{2} \sin \theta_{W}-\right. \\
& \left.\left(\alpha_{3}+\alpha_{9}\right) \cos \theta_{W}\right)+\frac{2 g^{2}}{v} \alpha_{11} \cos \theta_{W}\left[k_{\mu} q_{\nu}-r_{\mu} k_{\nu}\right]-\frac{2 g^{2}}{v} \alpha_{11} \sin ^{2} \theta_{W} \sec \theta_{W}\left[k^{2} g_{\mu \nu}-\right. \\
& \left.k_{\mu} q_{\nu}+r_{\mu} r_{\nu}\right]+\frac{g^{2}}{v} \alpha_{12} \sec \theta_{W}\left[k^{2} g_{\mu \nu}-k_{\mu} q_{\nu}+r_{\mu} r_{\nu}+q_{\mu} q_{\nu}\right]-\frac{2 g^{2}}{v} \alpha_{13} \sec \theta_{W} q_{\mu} q_{\nu} \\
& \mathrm{I}_{\pi^{+} W_{\mu}^{-} A_{\nu}}=-\frac{g^{2} v}{2} \sin ^{2} \theta_{W} g_{\mu \nu}-\frac{2 g^{2}}{v}\left[(q . r) g_{\mu \nu}-q_{\mu} r_{\nu}\right]\left(\alpha_{1} \cos \theta_{W}+\alpha_{8} \sin \theta_{W}\right)-\frac{2 g^{2}}{v}\left[(k . q) g_{\mu \nu}\right. \\
& \left.-q_{\mu} k_{\nu}\right]\left(\alpha_{2} \cos \theta_{W}+\left(\alpha_{3}+\alpha_{9}\right) \sin \theta_{W}\right)+\frac{2 g^{2}}{v} \alpha_{11} \sin \theta_{W}\left[k^{2} g_{\mu \nu}-k_{\nu} r_{\mu}+r_{\mu} r_{\nu}\right] \\
& \mathrm{I}_{W_{\mu}^{-} W_{\nu}^{+} Z_{\lambda}^{0}}=i g \cos \theta_{W}\left(1+\alpha_{3} g^{2} \sec ^{2} \theta_{W}\right)\left[(k-r)_{\lambda} g_{\mu \nu}+(r-q)_{\mu} g_{\nu \lambda}+(q-k)_{\nu} g_{\mu \lambda}\right]+i g^{2}\left\{g \alpha_{3}\right. \\
& \left.\sin \theta_{W} \operatorname{tg} \theta_{W}+g\left(\alpha_{2}-\alpha_{1}\right) \sin \theta_{W}+g\left(\alpha_{8}-\alpha_{9}\right) \cos \theta_{W}-\alpha_{13} g \sec \theta_{W}\right\}\left[q_{\mu} g_{\nu \lambda}-q_{\nu}\right. \\
& \left.g_{\mu \lambda}\right]+i g^{2}\left\{-g \alpha_{11} \sin \theta_{W} \operatorname{tg} \theta_{W}+\frac{\alpha_{12}}{2} g \sec \theta_{W}\right\}\left[k_{\mu} g_{\nu \lambda}-r_{\nu} g_{\mu \lambda}\right] \\
& \mathrm{I}_{W_{\mu}^{-} W_{\nu}^{+} A_{\lambda}}=i g \sin \theta_{W}\left[(k-r)_{\lambda} g_{\mu \nu}+(r-q)_{\mu} g_{\nu \lambda}+(q-k)_{\nu} g_{\mu \lambda}\right]+i g^{3}\left\{-\alpha_{3} \sin \theta_{W}+\left(\alpha_{1}-\right.\right.
\end{aligned}
$$




$$
\begin{aligned}
& \left.\left.\alpha_{2}\right) \cos \theta_{W}+\left(\alpha_{8}-\alpha_{9}\right) \sin \theta_{W}\right\}\left[q_{\mu} g_{\nu \lambda}-q_{\nu} g_{\mu \lambda}\right]+i g^{3} \alpha_{11} \sin \theta_{W}\left[k_{\mu} g_{\nu \lambda}\right. \\
& \left.-r_{\nu} g_{\mu \lambda}\right]
\end{aligned}
$$

iii) Here, we write the Feynman rules corresponding to the different vertices with 4 gauge electroweak bosons $\left(\mathrm{I}_{V_{1} V_{2} V_{3} V_{4}}\right)$, shown in Figure 3 .

$$
\begin{aligned}
& \mathrm{I}_{W_{\mu}^{-} W_{\nu}^{-} W_{\lambda}^{+} W_{\rho}^{+}}=i g^{2}\left\{1+g^{2}\left(\alpha_{4}-\alpha_{8}\right)+2 g^{2}\left(\alpha_{3}+\alpha_{9}+\alpha_{13}\right)\left[2 g_{\mu \nu} g_{\lambda \rho}-g_{\mu \lambda} g_{\nu \rho}-g_{\mu \rho} g_{\nu \lambda}\right]+2 i g^{4}\right. \\
& \left(\alpha_{4}+\alpha_{5}\right)\left[g_{\mu \lambda} g_{\nu \rho}+g_{\mu \rho} g_{\nu \lambda}\right] \\
& \mathrm{I}_{Z_{\mu}^{0} Z_{\nu}^{0} W_{\lambda}^{-} W_{\rho}^{+}}=i g^{2}\left\{-\cos ^{2} \theta_{W}-2 g^{2} \alpha_{3}+g^{2} \sec ^{2} \theta_{W}\left(\alpha_{5}+\alpha_{7}\right)\right\}\left[2 g_{\mu \nu} g_{\lambda \rho}-g_{\mu \lambda} g_{\nu \rho}-g_{\mu \rho} g_{\nu \lambda}\right]+i g^{4} \\
& \left\{\left(\alpha_{4}+\alpha_{5}+\alpha_{6}+\alpha_{7}\right) \sec ^{2} \theta_{W}+\alpha_{12} \operatorname{tg}^{2} \theta_{W}-\alpha_{11} \sin ^{2} \theta_{W} \operatorname{tg}^{2} \theta_{W}\right\}\left[g_{\mu \lambda} g_{\nu \rho}+g_{\mu \rho} g_{\nu \lambda}\right] \\
& \mathrm{I}_{A_{\mu} Z_{\nu}^{0} W_{\lambda}^{-} W_{\rho}^{+}}=-i g^{2} \sin \theta_{W} \cos \theta_{W}\left[1+g^{2} \sec ^{2} \theta_{W} \alpha_{3}\right]\left[2 g_{\mu \nu} g_{\lambda \rho}-g_{\mu \lambda} g_{\nu \rho}-g_{\mu \rho} g_{\nu \lambda}\right]+i g^{4}\left\{\sin ^{2} \theta_{W}\right. \\
& \left.\operatorname{tg} \theta_{W} \alpha_{11}-\frac{1}{2} \operatorname{tg} \theta_{W} \alpha_{12}\right\}\left[g_{\mu \lambda} g_{\nu \rho}+g_{\mu \rho} g_{\nu \lambda}\right] \\
& \mathrm{I}_{A_{\mu} A_{\nu} W_{\lambda}^{-} W_{\rho}^{+}}=-i g^{2}\left\{\sin ^{2} \theta_{W}\left[2 g_{\mu \nu} g_{\rho \lambda}-g_{\mu \lambda} g_{\nu \rho}-g_{\mu \rho} g_{\nu \lambda}\right]+g^{2} \sin ^{2} \theta_{W} \alpha_{11}\left[g_{\mu \lambda} g_{\nu \rho}+g_{\mu \rho} g_{\nu \lambda}\right]\right\} \\
& \mathrm{I}_{Z_{\mu}^{0} Z_{\nu}^{0} Z_{\lambda}^{0} Z_{\rho}^{0}}=2 i \sec ^{4} \theta_{W} g^{4}\left[\left(\alpha_{4}+\alpha_{5}\right)+2\left(\alpha_{6}+\alpha_{7}+\alpha_{10}\right)\right]\left[g_{\mu \nu} g_{\lambda \rho}+g_{\mu \lambda} g_{\nu \rho}+g_{\mu \rho} g_{\nu \lambda}\right]
\end{aligned}
$$




\section{References}

[1] S. L. Glashow, Nucl. Phys. 22 (1961) 579

S. Weinberg, Phys. Rev. Lett. 19 (1967) 1264

A. Salam, Proc. 8th Nobel Symp., ed. N. Svartholm, p. 367, Stockholm, Almqvist y Wiksells (1968)

[2] H.Haber and G. Kane, Phys. Rep. 117 (1985) 75

[3] S.Weinberg, Phys. Rev. D19 (1979) 1277

S.Dimopoulos and L.Susskind, Nucl. Phys. B155 (1979) 237

E.Farhi and L.Susskind, Phys. Rep. 74 (1981) 277

[4] A. Dobado and M.J. Herrero, Phys. Lett. B228 (1989) 495 and B233 (1989) 505

J.Donoghue and C. Ramírez, Phys. Lett. B234 (1990) 361

[5] B.Holdom and J. Terning, Phys.Lett. B247 (1990) 88

A. Dobado, D. Espriu and M.J. Herrero, Phys.Lett. B255 (1991) 405

M. Golden and L. Randall, Nucl. Phys. B361 (1991) 3

[6] S Weinberg, Physica A 96 (1979) 327

J.Gasser and H.Leutwyler, Ann. of Phys. 158 (1984) 142, Nucl. Phys. B250 (1985) 465 y 517

[7] P. Sikivie et al., Nucl. Phys. B173 (1980) 189

M. S. Chanowitz, M. Golden and H. Georgi, Phys.Rev. D36 (1987)1490

[8] A. Dobado, M.J. Herrero and J. Terrón, Z. Phys. C50 (1991) 205; Z. Phys. C50 (1991) 465 and Proceedings of the Large Hadron Collider Workshop, Aachen, 1990, Vol II, (G. Jarlskog and D. Rein, Geneva, 1990)

J.Bagger, S.Dawson y G.Valencia, Nucl.Phys. B399 (1993) 364

J. Bagger, V. Barger, K. Cheung, T. Han, G.A. Ladinsky, R. Rosenfeld y C.P. Yuan, Phys. Rev. D49 (1994) 1246

I.Jossa, F.Pauss and T.Rodrigo, Large Hadron Collider Workshop Aachen (1990), (1990), Vol II (Geneva 1990) 796.

[9] J.M. Cornwall, D.N. Levin and G. Tiktopoulos, Phys. Rev. D10 (1974) 1145

C.E. Vayonakis, Lett. Nuovo Cim.17(1976) 383

B.W. Lee, C. Quigg and H. Thacker, Phys. Rev. D16 (1977) 1519

M.S. Chanowitz and M.K. Gaillard, Nucl. Phys. 261 (1985)379 
[10] A. Dobado J. R. Peláez Nucl. Phys. B425 (1994) 110; Phys. Lett.B329(1994)469 (Addendum, ibid, B335 (1994) 554)

[11] Tran N. Truong, Phys. Rev. D61 (1988)2526

A. Dobado, M.J. Herrero and J.N. Truong, Phys. Lett. B235 (1990) 134

T.N.Truong, Phys. Rev. Lett. 67 (1991)2260; A. Dobado and J.R. Peláez, Phys. Rev. D47(1992) 4883

[12] C.J.C. Im, Phys. Lett. B281 (1992)357; A. Dobado and J.R. Peláez, Phys. Lett. B286 (1992)136

[13] T.Appelquist C.Bernard, Phys. Rev. D22 (1980) 200

A.Longhitano Phys. Rev. D22 (1980) 1166; Nucl. Phys. B188 (1981) 118

[14] F. Feruglio in the $2^{\text {nd }}$ Nat. Seminar of Th. Physics, Parma, Sept. 1992. $\mathrm{DFPD} 92 / \mathrm{TH} / 50$

[15] C.Weizsaker and E.J.Williams, Z. Phys. 88 (1934) 612

[16] S. Dawson, Nucl. Phys. B249 (1985) 42

[17] E.W.N.Glover and J.J.Van deer Bij, Nucl. Phys. B321 (1989) 561

[18] A.Falk, M.Luke and E.Simmons, Nucl. Phys. B365 (1991) 523

[19] A.Dobado and M.Urdiales, Phys. Lett. B292 (1992) 128

[20] M.Herrero and E.Ruiz Morales, Nucl. Phys. B418 (1994) 431; FTUAM 94/11, hepph/9411207 to appear in Nucl. Phys. B (1995)

[21] A.Dobado, J.R.Peláez and M.T.Urdiales, Contributed paper to the 27th International Conference in High Energy Physics (Glasgow , July 1994) hep-ph / 9407384, and work in preparation.

[22] E.Eitchen et.al., Rev. Mod. Phys. 56 (1984) 579

[23] G.P. Lepage, J. Comput. Phys. 27 (1978) 192

[24] A.D. Martin, R.G Roberts and W.J. Stirling, Phys. Lett. 306B (1993) 145 and Phys. Lett. bf 309B (1993) 492

[25] M. Glück, E. Reya and A. Vogt, Z. Phys. C48 (1990) 471, Z. Phys. C53 (1992) 127 and Phys. Lett. 306B (1993) 391 
[26] J. Terrón, private communication

[27] Particle Data Group, J.J.Hernández et.al., Review of particle properties, Phys. Lett. B239 (1990) 1 


\section{Table Captions}

Table 1. In this Table we display the relations between different sets of chiral Lagrangian parameters. They correspond to those used by Longhitano [13], Feruglio [14] and Dobado et.al. [5], in columns 1, 2 and 3, respectively.

Table 2. In this table we show the sensitivity functions $s_{k}^{(Z Z)}$ and $s_{k}^{(W Z)}$ associated to each $\alpha_{k}(k=0,1,2,3,4,5)$ parameter. They were calculated with the minimal cuts and under the hypothesis of linear behaviour of $N^{(i)}(\alpha ; c)$ with respect to each $\alpha_{k}$.

Table 3. Optimal cuts to calculate the statistical significance $r_{k}^{(i)}(c)$ for typical positive and negative $\alpha_{k}$ values $\left(\alpha_{k}= \pm 0.005\right)$ in the $Z^{0} Z^{0}$ or $W^{ \pm} Z^{0}$ final states. The first entry in column 3 refers to the minimum invariant mass and the second to the minimum allowed $p_{T Z}$, both them given in GeV. The total number of events in the Zero Model and with the typical chosen $\alpha_{k}$ obtained after having applied the optimal cuts, are represented in columns 4 and 5. They correspond to 1 year of running for the $L H C$ and a maximal rapidity of 2.5 for the final bosons.

Table 4 . Here, we shown a comparison between the statistical significance function $r_{k}^{(i)}(c)$ given by the typical $\alpha_{k}$ in $Z^{0} Z^{0}$ or $W^{ \pm} Z^{0}$ final state, for the minimal and the optimal cuts. In both cases, we have fixed the maximal rapidity cut of 2.5 for two gauge final bosons, and 1 year of working time for the $L H C$.

Table 5. In column 3, we display the statistical errors, $\left(\Delta \alpha_{k}\right)_{\text {stat. }}$, corresponding to each $\alpha_{k}$ parameter in the $Z^{0} Z^{0}$ or $W^{ \pm} Z^{0}$ channels. They were obtained with the optimal cuts (column 1) and under the assumption of linear dependence of the total number of events on $\alpha_{k}$.

Table 6. Comparison of the total number of $Z^{0} Z^{0}$ or $W^{ \pm} Z^{0}$ events obtained in the Zero Model applying the optimal cuts and choosing different structure functions: $E H L Q$, $M R S D-$ and $G R V H O$. In the last three columns, we represent the statistical significance to estimate these imprecisions, $r_{\text {struc. }}^{(i)}(c)$ eq. (22), between EHLQ and MRSD- (column 6), EHLQ and GRVHO (7 column) and $M R S D-$ and GRVHO (8 column). These uncertainties are called, respectively, $r_{12}^{(i)}, r_{13}^{(i)}$ and $r_{23}^{(i)}$. In all these computations we have taken $y_{1 \max }=y_{2 \max }=2.5$ and an integrated luminosity for the $L H C$ of $3 \times 10^{5} \mathrm{pb}^{-1}$.

Table 7. In this Table we represent the total number of $Z^{0} Z^{0}$ or $W^{ \pm} Z^{0}$ pairs obtained at $L H C$ using the optimal cuts and setting $y_{1 \max }=y_{2 \max }=2.5$ and an integrated luminosity for the $L H C$ of $3 \times 10^{5} p b^{-1}$. In columns 2 and 3 we display the results in the Zero Model 
and with constant $g$ and $g^{\prime}$ couplings. Again in columns 4 and 5 we show the total number of $Z^{0} Z^{0}$ or $W^{ \pm} Z^{0}$ events but in the Running Zero Model and with $g(\sqrt{\hat{s}})$ and $g^{\prime}(\sqrt{\hat{s}})$, eq.(28). In the last two columns we display a measure of the statistical significance that corresponds to include the dependence on the energy of the parameters and couplings, given by the $r_{\text {run. }}^{(Z Z)}(c)$ or $r_{\text {run. }}^{(W Z)}(c)$ functions, eq.(29). 


\section{Figure Captions}

Figure 8. Propagators of the $\pi^{ \pm}$and $W^{ \pm}$.

Figure 2. Vertices with three gauge bosons or two gauge bosons and one $G B$, obtained from $\mathcal{L}_{\mathrm{NLSM}}$ eq.(7).

Figure 0 . Different four gauge boson vertices that appear in the theory described by the lagrangian $\mathcal{L}_{\mathrm{NLSM}}$ eq.(7).

Figure 1 . Different diagrams to one loop order contributing to $g g \rightarrow Z^{0} Z^{0}$ cross section, in the $S M$ without a Higgs boson.

Figure 5. We display the diagrams at tree level order that contribute to the cross section $q \bar{q} \rightarrow Z^{0} Z^{0}$ in the framework of chiral perturbation theory (with $\mathcal{L}_{\mathrm{NLSM}}$ ). In this case we obtain the same result as in the $S M$ without a Higgs boson.

Figure 6. We show the only diagram contributing to the different $Z^{0} Z^{0} \rightarrow Z^{0} Z^{0}$ helicity amplitudes obtained with $\mathcal{L}_{\mathrm{NLSM}}$ eq.(7), at tree level order.

Figure 7. The scattering amplitudes for the process $W^{+} W^{-} \rightarrow Z^{0} Z^{0}$, calculated with $\mathcal{L}_{\text {NLSM }}$ eq.(7) at tree level order in the Landau gauge, receive contribution from all these channels.

Figure 8. The cross sections $q \bar{q}^{\prime} \rightarrow W^{ \pm} Z^{0}$ are calculated adding the contribution of the diagrams displayed in this Figure. They are obtained with $\mathcal{L}_{\text {NLSM }}$ eq.(7) at tree level order and in the Landau gauge. The initial quarks are supposed to be massless.

Figure 9. Different diagrams contributing to the $W^{ \pm} Z^{0} \rightarrow W^{ \pm} Z^{0}$ scattering amplitudes. They are calculated with $\mathcal{L}_{\mathrm{NLSM}}$ eq.(7) at tree level order and in the Landau gauge.

Figure 10. The scattering amplitudes $W^{ \pm} \gamma \rightarrow W^{ \pm} Z^{0}$ are obtained with the Lagrangian given in eq.(7) adding the contribution of the diagrams displayed in this Figure. The calculation is made at tree level order and fixing the Landau gauge.

Figure 11. This graphic represents the sensitivity of the $L H C$ to the $\alpha_{0}$ parameter in $Z^{0} Z^{0}$ and $W^{ \pm} Z^{0}$ channels. We display the total number of $Z^{0} Z^{0}$ (solid line) and $W^{ \pm} Z^{0}$ (dashed line) events obtained in the $L H C$, for different values of the $\alpha_{0}$ parameter. The kinematical cuts are the minimal ones. The other $\alpha_{k}$ parameters, apart from $\alpha_{0}$, have been set to zero. 
Figure 12. This Figure represents the sensitivity function to $\alpha_{1}$ parameter for the $L H C$, using the same kinematical cuts as those in Figure 11. We display the total $N^{(Z Z)}$ (solid line) and $N^{(W Z)}$ (dashed line) events obtained versus $\alpha_{1}$ values.

Figure 13. This Figure represents the sensitivity function to $\alpha_{2}$ parameter for the $L H C$. As in Figures 11 and 12 the total number of $Z^{0} Z^{0}$ and $W^{ \pm} Z^{0}$ events versus $\alpha_{2}$ values.

Figure 14. This Figure represents the sensitivity function to $\alpha_{3}$ parameter for the $L H C$, using the same kinematical cuts as in previous Figures. We show the total $N^{(Z Z)}$ (solid line) and $N^{(W Z)}$ (dashed line) events versus $\alpha_{3}$.

Figure 15. This graphic represents the sensitivity of the $L H C$ to the $\alpha_{4}$ parameter in the $Z^{0} Z^{0}$ and $W^{ \pm} Z^{0}$ channels. We display, like in Figures 11, 12, 13 and 14, the total number of $Z^{0} Z^{0}$ (solid line) and $W^{ \pm} Z^{0}$ (dashed line) events obtained at $L H C$, for different values of the $\alpha_{4}$ parameter, using the same kinematical cuts as in previous Figures.

Figure 16. Sensitivity function to $\alpha_{5}$ parameter for the $L H C$. We have used the same kinematical cuts as in previous Figures. We display the total $N^{(Z Z)}$ (solid line) and $N^{(W Z)}$ (dashed line) events obtained versus $\alpha_{5}$ values, as we did in figures 11 to 15 .

Figure 17. In this Figure we study the variation of the total number of $Z^{0} Z^{0}$ (solid line) and $W^{ \pm} Z^{0}$ (dashed line) events with the cut on the minimal invariant mass, $\sqrt{\hat{s}_{\min }}$. We have set all the $\alpha_{k}$ parameters to their values in the Zero Model and the cuts $p_{T Z_{\min }}, y_{1 \max }$ and $y_{2 \max }$ to their minimal values.

Figure 18. As in Figure 17, we display the behaviour of the total number of $Z^{0} Z^{0}$ (solid line) and $W^{ \pm} Z^{0}$ (dashed line) events in the Zero Model versus the cut in the minimal transversal momentum of the $Z^{0}$ boson. We have taken the other kinematical bounds as their minimal values.

Figure 19. Here we reflect the dependence of the total number of $Z^{0} Z^{0}$ and $W^{ \pm} Z^{0}$ events on the maximal rapidity bounds of final gauge bosons, $y_{1 \max }=y_{2 \max }$. We work in the Zero Model and fix the minimal cuts in invariant mass and transversal moment. The solid line corresponds to the $Z^{0} Z^{0}$ final state and the dashed one to the produced $W^{ \pm} Z^{0}$ pairs.

Figure 20. Statistical significance corresponding to the $\alpha_{0}$ parameter. We display the $r_{0}$ function eq.(18) for different values of $\alpha_{0}$ with respect to the Zero Model. The solid line represents the $Z^{0} Z^{0}$ final state and the dashed line the $W^{ \pm} Z^{0}$. We use the same kinematical cuts as in Figures 11 to 16. 
Figure 21. Statistical significance corresponding to the $\alpha_{1}$ parameter. We show as in Figure 20, the $r_{1}^{(Z Z)}$ (solid) eq.(18) and $r_{1}^{(W Z)}$ (dashed) functions with respect to the Zero Model versus these $\alpha_{1}$ values. We use the same kinematical cuts as in Figures 11 to 16.

Figure 22. This Figure represents the statistical significance for some values of $\alpha_{2}$ with respect to the Zero Model. We display as in Figures 20 and 21, and for the same kinematical cuts, the $r_{2}^{(Z Z)}$ and $r_{2}^{(W Z)}$ eq.(18) functions.

Figure 23. This Figure reflects the statistical significance of $\alpha_{3}$. We show the $r_{k}$ eq.(18) functions for $Z^{0} Z^{0}$ (solid) and $W^{ \pm} Z^{0}$ (dashed) with respect to the Zero Model versus $\alpha_{3}$ values, using the same kinematical cuts as in previous Figures.

Figure 24. Statistical significance corresponding to the $\alpha_{4}$ parameter. As in Figures 20 to 23 we show the $r_{4}^{(Z Z)}$ (solid) (eq. 18) and $r_{4}^{(W Z)}$ (dashed) with respect to the Zero Model versus the $\alpha_{4}$ values. We have used the same kinematical cuts as in previous figures.

Figure 25. The same as in Figures 20 to 24 for the $\alpha_{5}$ parameter. 
Table 1:

\begin{tabular}{|c|c|c|}
\hline \hline$\alpha_{0}$ & $a_{0} / g^{2}$ & $\delta \beta / g^{2}$ \\
\hline$\alpha_{1}$ & $g^{\prime} / g a_{1}$ & $L_{10} \operatorname{tg} \theta_{W}$ \\
\hline$\alpha_{2}$ & $g^{\prime} / g a_{2}$ & $-L_{9 R} / 2 \operatorname{tg} \theta_{W}$ \\
\hline$\alpha_{3}$ & $-a_{3}$ & $-L_{9 L} / 2$ \\
\hline$\alpha_{4}$ & $a_{4}$ & $L_{2}$ \\
\hline$\alpha_{5}$ & $a_{5}$ & $L_{1}$ \\
\hline$\alpha_{6}$ & $a_{6}$ & - \\
\hline$\alpha_{7}$ & $a_{7}$ & - \\
\hline$\alpha_{8}$ & $-a_{8}$ & - \\
\hline$\alpha_{9}$ & $-a_{9}$ & - \\
\hline$\alpha_{10}$ & $2 a_{10}$ & - \\
\hline$\alpha_{11}$ & $a_{11}$ & - \\
\hline$\alpha_{12}$ & $2 a_{12}$ & - \\
\hline$\alpha_{13}$ & $a_{13}$ & - \\
\hline
\end{tabular}

Table 2:

\begin{tabular}{|r|r|r|}
\hline \hline$\alpha_{k}$ & \multicolumn{1}{|c|}{$s_{k}^{(Z Z)}\left(c^{\mathrm{min}}\right)$} & $s_{k}^{(W Z)}\left(c^{\mathrm{min}}\right)$ \\
\hline$\alpha_{0}$ & -899.54 & 2779.89 \\
\hline$\alpha_{1}$ & 1241.27 & -5408.20 \\
\hline$\alpha_{2}$ & -1481.65 & 6150.98 \\
\hline$\alpha_{3}$ & 6751.24 & -48366.79 \\
\hline$\alpha_{4}$ & 2445.43 & -18981.46 \\
\hline$\alpha_{5}$ & 5924.90 & -20142.33 \\
\hline
\end{tabular}


Table 3:

\begin{tabular}{|l|c|c|c|c|}
\hline \hline$\alpha_{k}$ value & Channel & $c^{\text {op }}=\left(\sqrt{\hat{s}_{\min }^{\text {op }}}, p_{T \min }^{\text {op }}\right)$ & $N^{(i)}\left(\alpha^{0} ; c^{\text {op }}\right)$ & $N^{(i)}\left(\alpha ; c^{\text {op }}\right)$ \\
\hline$\alpha_{3}=-0.005$ & $Z^{0} Z^{0}$ & $(200,10)$ & 16152.55 & 16119.28 \\
\hline$\alpha_{3}=-0.005$ & $W^{ \pm} Z^{0}$ & $(200,300)$ & 522.59 & 581.41 \\
\hline$\alpha_{3}=0.005$ & $Z^{0} Z^{0}$ & $(200,10)$ & 16152.55 & 16186.47 \\
\hline$\alpha_{3}=0.005$ & $W^{ \pm} Z^{0}$ & $(200,200)$ & 2268.62 & 2160.74 \\
\hline$\alpha_{4}=-0.005$ & $W^{ \pm} Z^{0}$ & $(1150,500)$ & 32.29 & 66.48 \\
\hline$\alpha_{4}=0.005$ & $Z^{0} Z^{0}$ & $(1150,400)$ & 13.50 & 20.01 \\
\hline$\alpha_{5}=-0.005$ & $W^{ \pm} Z^{0}$ & $(1150,400)$ & 61.56 & 85.78 \\
\hline$\alpha_{5}=0.005$ & $Z^{0} Z^{0}$ & $(1150,400)$ & 13.50 & 28.70 \\
\hline
\end{tabular}

Table 4:

\begin{tabular}{|l|c|c|c|}
\hline \hline$\alpha_{k}$ value & Channel & $r_{k}\left(\alpha ; c^{\mathrm{min}}\right)$ & $r_{k}\left(\alpha ; c^{\text {op }}\right)$ \\
\hline$\alpha_{3}=-0.005$ & $Z^{0} Z^{0}$ & 0.26 & 0.26 \\
\hline$\alpha_{3}=-0.005$ & $W^{ \pm} Z^{0}$ & 1.01 & 2.57 \\
\hline$\alpha_{3}=0.005$ & $Z^{0} Z^{0}$ & 0.27 & 0.27 \\
\hline$\alpha_{3}=0.005$ & $W^{ \pm} Z^{0}$ & 0.51 & 2.27 \\
\hline$\alpha_{4}=-0.005$ & $W^{ \pm} Z^{0}$ & 0.45 & 6.02 \\
\hline$\alpha_{4}=0.005$ & $Z^{0} Z^{0}$ & 0.18 & 1.77 \\
\hline$\alpha_{5}=-0.005$ & $W^{ \pm} Z^{0}$ & 0.39 & 3.09 \\
\hline$\alpha_{5}=0.005$ & $Z^{0} Z^{0}$ & 0.37 & 4.14 \\
\hline
\end{tabular}


Table 5:

\begin{tabular}{|c|c|c|}
\hline \hline$c^{\mathrm{op}}=\left(\sqrt{\hat{s}_{\text {min }}^{\mathrm{op}}}, p_{T \text { min }}^{\text {op }}\right)$ & Channel & $\left(\Delta \alpha_{k}\right)_{\text {stat. }}$ \\
\hline$(200,300)$ & $W^{ \pm} Z^{0}$ & $\left(\Delta \alpha_{3}\right)_{\text {stat. }}=1.95 \times 10^{-3}$ \\
\hline$(200,200)$ & $W^{ \pm} Z^{0}$ & $\left(\Delta \alpha_{3}\right)_{\text {stat. }}=2.20 \times 10^{-3}$ \\
\hline$(1150,500)$ & $W^{ \pm} Z^{0}$ & $\left(\Delta \alpha_{4}\right)_{\text {stat. }}=8.31 \times 10^{-4}$ \\
\hline$(1150,400)$ & $Z^{0} Z^{0}$ & $\left(\Delta \alpha_{4}\right)_{\text {stat. }}=2.82 \times 10^{-3}$ \\
\hline$(1150,400)$ & $W^{ \pm} Z^{0}$ & $\left(\Delta \alpha_{5}\right)_{\text {stat. }}=1.62 \times 10^{-3}$ \\
\hline$(1150,400)$ & $Z^{0} Z^{0}$ & $\left(\Delta \alpha_{5}\right)_{\text {stat. }}=1.21 \times 10^{-3}$ \\
\hline
\end{tabular}


Table 6:

\begin{tabular}{|c|c|c|c|c|c|c|c|}
\hline \hline Channel & $\begin{array}{c}\text { Optimal cuts } \\
\left(\hat{\hat{s}}_{\min }^{\mathrm{op}}, p_{\text {min }}^{\text {op }}\right)\end{array}$ & $\begin{array}{c}N^{(i)}\left(\alpha^{0} ; c^{\mathrm{op}}\right) \\
E H L Q(\text { set II })\end{array}$ & $\begin{array}{c}N^{(i)}\left(\alpha^{0} ; c^{\mathrm{op}}\right) \\
M R S-D^{\prime}\end{array}$ & $\begin{array}{c}N^{(i)}\left(\alpha^{0} ; c^{\mathrm{op}}\right) \\
G R V H O\end{array}$ & $r_{12}^{(i)}$ & $r_{13}^{(i)}$ & $r_{23}^{(i)}$ \\
\hline$Z Z$ & $(200,10)$ & 16113.31 & 17242.93 & 16617.27 & 8.90 & 3.97 & 4.76 \\
\hline$W Z$ & $(200,200)$ & 2298.57 & 2526.75 & 2452.56 & 4.76 & 3.21 & 1.48 \\
\hline$W Z$ & $(200,300)$ & 535.80 & 594.34 & 578.75 & 2.53 & 1.86 & 0.64 \\
\hline$W Z$ & $(1150,500)$ & 36.02 & 40.79 & 39.97 & 0.79 & 0.66 & 0.13 \\
\hline$Z Z$ & $(1150,400)$ & 14.92 & 15.88 & 15.39 & 0.25 & 0.12 & 0.12 \\
\hline$W Z$ & $(1150,400)$ & 68.02 & 76.72 & 74.78 & 1.05 & 0.82 & 0.22 \\
\hline
\end{tabular}

Table 7:

\begin{tabular}{|c|c|c|c|c|c|c|}
\hline Optimal cuts & \multicolumn{2}{|c|}{ No running } & \multicolumn{2}{|c|}{ Running } & \multicolumn{2}{|c|}{$r_{\text {run. }}^{(i)}$} \\
\hline$\left(\sqrt{\hat{s}_{\min }}\right)$ & $N^{(Z Z)}\left(\alpha^{0} ; c^{\mathrm{op}}\right)$ & $N^{(W Z)}\left(\alpha^{0} ; c^{\mathrm{op}}\right)$ & $N^{(Z Z)}\left(\alpha^{0} ; c^{\mathrm{op}}\right)$ & $N^{(W Z)}\left(\alpha^{0} ; c^{\mathrm{op}}\right)$ & $r_{\text {run. }}^{(Z Z)}$ & $r_{\text {run. }}^{(W Z Z)}$ \\
\hline$(200,10)$ & 16149.13 & 91141.49 & 15355.82 & 86149.13 & 6.24 & 16.54 \\
\hline$(200,200)$ & - & 2281.63 & - & 2093.83 & - & 3.93 \\
\hline$(200,300)$ & - & 532.33 & - & 505.18 & - & 1.18 \\
\hline$(1150,500)$ & - & 36.01 & - & 45.75 & - & 1.62 \\
\hline$(1150,400)$ & 14.91 & 68.01 & 11.28 & 78.07 & 0.94 & 1.22 \\
\hline
\end{tabular}


Figure 1: 
Figure 2: 
Figure 3: 
Figure 4:

Figure 5: 
Figure 6: 
Figure 7: 
Figure 8:

Figure 9: 
Figure 10: 
Figure 11:

Figure 12:

Figure 13:

Figure 14: 
Figure 15:

Figure 16: 
Figure 17:

Figure 18: 
Figure 19: 
Figure 20:

Figure 21: 
Figure 22: 
Figure 23:

Figure 24: 
Figure 25: 